\title{
Solving the Steiner Tree Problem with few Terminals
}

\author{
Fichte Johannes K. ${ }^{1}$, Hecher Markus*2, and Schidler André ${ }^{2}$ \\ ${ }^{1} \mathrm{TU}$ Dresden \\ ${ }^{2} \mathrm{TU}$ Wien
}

November 10, 2020

\begin{abstract}
The Steiner tree problem is a well-known problem in network design, routing, and VLSI design. Given a graph, edge costs, and a set of dedicated vertices (terminals), the Steiner tree problem asks to output a sub-graph that connects all terminals at minimum cost. A state-of-theart algorithm to solve the Steiner tree problem by means of dynamic programming is the Dijkstra-Steiner algorithm. The algorithm builds a Steiner tree of the entire instance by systematically searching for smaller instances, based on subsets of the terminals, and combining Steiner trees for these smaller instances. The search heavily relies on a guiding heuristic function in order to prune the search space. However, to ensure correctness, this algorithm allows only for limited heuristic functions, namely, those that satisfy a so-called consistency condition.

In this paper, we enhance the Dijkstra-Steiner algorithm and establish a revisited algorithm, called $\mathrm{DS}^{*}$. The $\mathrm{DS}^{*}$ algorithm allows for arbitrary lower bounds as heuristics relaxing the previous condition on the heuristic function. Notably, we can now use linear programming based lower bounds. Further, we capture new requirements for a heuristic function in a condition, which we call admissibility. We show that admissibility is indeed weaker than consistency and establish
\end{abstract}

${ }^{*}$ Hecher is also affiliated with the University of Potsdam, Germany. 
correctness of the $\mathrm{DS}^{*}$ algorithm when using an admissible heuristic function. We implement $\mathrm{DS}^{*}$ and combine it with modern preprocessing, resulting in an open-source solver (DS* Solve). Finally, we compare its performance on standard benchmarks and observe a competitive behavior.

\section{Introduction}

The term Steiner tree problem on graphs encompasses a class of graph problems that all ask to connect specific vertices of a graph, so-called terminals, at minimum cost. Connecting the vertices usually requires to select edges of a given graph that form a tree and the resulting solution is called a Steiner tree. While numerous different definitions exist [19], we consider the minimum Steiner tree problem (STP), where costs are defined by positive integers given at the edges. The total cost is simply the sum of costs of selected edges and one aims to minimize the total cost [21].

STP is long known to be computationally hard, i.e., NP-hard [25]. Despite the longevity and the large body of existing research, solving STP is still an active research topic $[13,20,23,30,31]$. Interest stems from applications in various fields such as the construction of evolutionary trees in phylogeny [17], network design [26], routing problems [15], and VLSI design [16]. Over time, many heuristic and exact solving algorithms and techniques have been developed [21]. One of the longest known algorithms for STP is the DreyfusWagner algorithm [8]. This algorithm implements dynamic programming with runtime bounded exponentially in the number of terminals and polynomially in the number of vertices. In theory, the Dreyfus-Wagner algorithm is fast as long as the number of terminals remains small. However, in practice, the involved constants are far from optimal and the runtime of the algorithm increases too quickly as the algorithm exhaustively enumerates all sub-solutions. This can be avoided and the runtime improved by replacing enumeration with graph search, resulting in the Dijkstra-Steiner algorithm [20]. Its underlying idea is similar to the well-known $\mathrm{A}^{*}$ algorithm [18]. One uses the Dijkstra's algorithm to navigate the search space and guide the search using a heuristic function. In practice, the heuristic function is crucial for the performance of the algorithm. Unfortunately, the heuristic needs to satisfy a strong condition, namely, providing consistent lower bounds, as otherwise correctness cannot be assured. Consistency guarantees that the lower bound is monotonous and the 
estimate will not decrease in later iterations of the Dijkstra-Steiner algorithm.

Contributions. In this paper, we revisit the Dijkstra-Steiner algorithm and enable heuristic functions for general lower bounds relaxing the previously known consistency condition. We formalize new requirements in a condition called admissibility. We prove that admissibility is weaker than consistency and allows for better heuristic functions. In particular, we can now employ linear programming based approximations as heuristics. We show that our approach still ensures correctness. We implement the new algorithm into a fully fledged solver, $D S^{*}$ Solve, for STP. Our solver complements the solving algorithm with modern preprocessing, upper bound heuristics, and local optimization. Finally, we present experimental results where we compare our solver to state-of-the-art STP solvers. Our experiments show that our revisited algorithm with the new heuristic significantly improves the runtime.

Algorithms and latest implementations. The Dreyfus-Wagner algorithm [8] was generalized by Erickson, Monma, and Veinott [11]. While the algorithm is exponential in the number of terminals with a basis of 3 , it was very recently implemented into the solver Pruned showing good performance on certain benchmarks [23]. The Dijkstra-Steiner algorithm 20] is implemented in the solvers Jagiellonian [28] and HSV [20]. We also implemented it into our solver to measure the difference with our revisited algorithm and heuristic. While our approach tackles instances with a limited number of terminals, there are also dynamic programming algorithms that employ low treewidth or rank [1,3]. The solvers wata_sigma [22], Tom [39], and FIT CTU [29] implement such algorithms and showed successful results in the PACE 2018 challenge. Another approach is branch-and-cut, which is based on linear programming using existing solvers for (mixed) integer linear programs (MILP). Various solvers are available that perform very well on arbitrary STP instances and can mainly be distinguished by the used linear programming model. Two notable solvers are mozartballs [13 and SCIP-Jack [14.

Challenges. In 2014, the 11th DIMACS implementation challenge was dedicated to different variants of the Steiner tree problem [24]. The 3rd Parameterized Algorithms and Computational Experiments challenge (PACE 2018) addressed the minimum Steiner tree problem and featured three tracks, namely, a) where instances were limited in the number of terminals, b) where instances were limited in the treewidth, and c) that allowed to submit heuristics [2]. Solvers in these competitions mainly used two different techniques: 

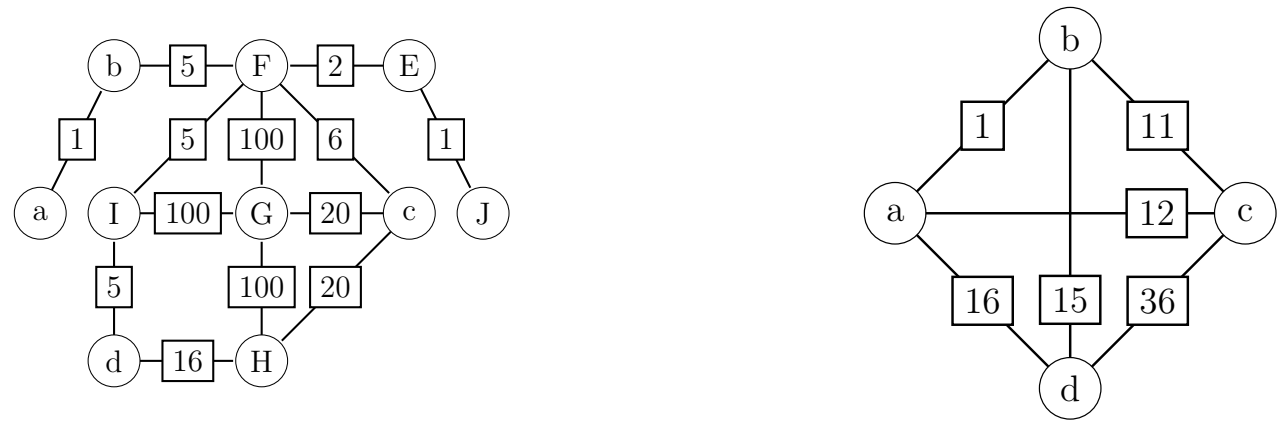

Figure 1: Example of a network $N$ (left), distance network $D_{N}(\{a, b, c, d\})$ (middle), and an $\operatorname{SMT}(N,\{a, b, c, d\})$ (right).

dynamic programming and branch-and-cut.

\section{Preliminaries}

We assume familiarity with standard notions in computational complexity 32 and let $\mathbb{N}$ be the set of positive integers.

Graphs, Networks, and Steiner Trees. For basic terminology on graphs, we refer to the literature [6]. In particular, we use $G=(V, E)$ to denote an undirected, connected graph, or graph for short, where $V$ is a set of vertices and $E \subseteq\{\{u, v\} \mid u, v \in V\}$ is a set of edges. Given graphs $G_{1}=\left(V_{1}, E_{1}\right)$ and $G_{2}=\left(V_{2}, E_{2}\right), G_{1}$ is a sub-graph of $G_{2}$ if $V_{1} \subseteq V_{2}$ and $E_{1} \subseteq E_{2}$. Given a graph $G=(V, E)$ and a vertex $u \in V$. Then, the set $\delta_{G}(u)$ of incident edges is given by $\delta_{G}(u):=\{\{u, v\} \mid\{u, v\} \in E\}$ and $\left|\delta_{G}(u)\right|$ refers to the degree of $u$. A tree is a graph that is acyclic and connected, i.e., for every pair of vertices of the graph there is a path between them. Further, we define an undirected network, or network for short, by $N:=(V, E, \sigma)$, where graph $(V, E)$ is connected and $\sigma: E \rightarrow \mathbb{N}$ is a total mapping, called edge costs, which assigns to each edge $e$ some integer, called cost of $e$. Let $N=(V, E, \sigma)$ be a network and let $G=\left(V^{\prime}, E^{\prime}\right)$ be a sub-graph of $(V, E)$. We let the costs of $G$ in $N$ be $c_{N}(G):=\sum_{e \in E^{\prime}} \sigma(e)$. Then, we denote by $d_{N}(u, v)$ the distance between two vertices $u \in V$ and $v \in V$ in $N$, i.e., $d_{N}(u, v)$ is the length of the shortest path from $u$ to $v$. Formally, $d_{N}(u, v):=\min \left\{c_{N}(P) \mid\right.$ $P$ is a connected sub-graph of $G$ that contains $u$ and $v\}$. Further, we define the distance network of $V^{\prime}$ for $N$ by $D_{N}\left(V^{\prime}\right):=\left(V^{\prime},\left\{\{u, v\} \mid u, v \in V^{\prime}\right\}, \sigma^{\prime}\right)$, where $\sigma^{\prime}(\{u, v\}):=d_{N}(u, v)$ for $u, v \in V^{\prime}$. 
Example 1. Figure 1 (left) illustrates network $N$, which we use throughout the paper. Circles represent vertices, lines are edges, and edge costs are given by the rectangles. Further, terminals are denoted by lowercase letters. Figure 1 (middle) provides the distance network $D_{N}(\{a, b, c, d\})$ for $N$.

An instance $\mathcal{I}$ of the minimum Steiner Tree Problem (STP) is of the form $\mathcal{I}:=(N, R)$, where $N=(V, E, \sigma)$ is a network and $R \subseteq V$ is a nonempty set of vertices, called terminals. A Steiner tree for $\mathcal{I}$ is a rooted tree $T:=\left(V^{\prime}, E^{\prime}, r\right)$, where $V^{\prime} \subseteq V, R \subseteq V^{\prime}, E^{\prime} \subseteq E$, and $r \in R$. We call $r$ the root. $\mathcal{T}(\mathcal{I})$ refers to the set of Steiner trees for $\mathcal{I}$. The tree $T$ is called an SMT (Steiner minimal tree), if $c_{N}(T)=\min _{T^{\prime} \in \mathcal{T}(\mathcal{I})} c_{N}\left(T^{\prime}\right)$. Let $\operatorname{smt}(\mathcal{I})$ be the set of SMTs for $\mathcal{I}$ and let $\operatorname{csmt}(\mathcal{I}):=c_{N}(T)$ for any $T \in \operatorname{smt}(\mathcal{I})$, called the csmt (Steiner minimal tree costs). The tree $T$ is a minimum spanning tree of network $N$, if $T$ is an SMT for $(N, V)$.

Example 2. Figure 1 (right) depicts the $S M T$ for our instance $\mathcal{I}=(N, R)$ from Example 1, where $N$ is given (left). The set $R$ of terminals consists of the vertices $R=\{a, b, c, d\}$ in lower case letters (left).

Dijkstra's algorithm and the $A^{*}$ algorithm. Given a network $N=$ $(V, E, \sigma)$. Dijkstra's algorithm is used to find the shortest path from vertex $u \in V$ to vertex $v \in V$. In the following, we briefly mention the ideas of this algorithm. For details, we refer to the literature [7]. Dijkstra's algorithm maintains a queue $Q$ and for each vertex $w \in V$ a distance $l(w)$ between vertices $u$ and $w$. Initially, $Q=\{u\}$ and the distances $l(\cdot)$ are assumed to be $\propto^{1}$, except for $l(u)$, where the distance is known to be 0 . In each iteration the algorithm removes the vertex $w$ from $Q$ that minimizes $l(w)$. Then, the algorithm expands $w$ : For each $\{w, x\} \in \delta_{(V, E)}(w)$, the value $l(w)+\sigma(\{w, x\})$ is computed. Whenever $l(w)+\sigma(\{w, x\})<l(x)$, vertex $x$ is added to $Q$ and the (smaller) distance $l(x) \leftarrow l(w)+\sigma(\{w, x\})$ is kept for $x$. Whenever a node $w$ is removed from $Q$, it holds that $l(w)=d_{N}(u, w)$. Therefore, the algorithm stops as soon as $v$ is removed from $Q$ and finds the distance in time $\mathcal{O}(|E|+|V| \cdot \log (|V|))[7]$.

The $A^{*}$ algorithm is an extension of Dijkstra's algorithm that uses a heuristic function $h: V \rightarrow \mathbb{N}$ to speed up the search. Assuming $h(x)$ is an estimate of the distance between $u$ and $x$, in each iteration of $A^{*}$, instead of removing vertex $w$ from $Q$, where $l(w)$ is minimal as in Dijkstra's algorithm, the $A^{*}$ algorithm removes $w$, where $l(w)+h(w)$ is minimal. We call $h$

\footnotetext{
${ }^{1}$ In this paper, we use $\infty$ as an abbreviation for $\Sigma_{e \in E} \sigma(e)$.
} 
- admissible, if $h(w) \leq d_{N}(w, v)$ for all $w \in V$, and

- consistent, if $h(w) \leq \sigma(\{w, y\})+h(y)$ for all $\{w, y\} \in E$.

Intuitively, admissibility of $h$ implies that $h$ does not "over-approximate", i.e., it provides a lower bound, and consistency of $h$ additionally establishes a form of triangle inequality for $h$. While $A^{*}$ is correct if $h$ is admissible, polynomial runtime can only be guaranteed if the heuristic is consistent [18].

\section{Solving the Steiner Tree Problem}

In this section, we describe our advancement to the Dijkstra-Steiner (DS) algorithm. DS combines ideas from the A* and Dreyfus-Wagner algorithms. We first discuss the Dreyfus-Wagner algorithm, followed by DS. Finally, we lift DS to more general heuristic functions, which provide a lower bound on the costs, and show correctness. For this section, we assume an STP instance $\mathcal{I}=(N, R)$ with network $N=(V, E, \sigma)$ and set $R$ of terminals, where $r \in R$ is an arbitrary terminal. The vertex $r$ is used as the root of the resulting SMT.

\subsection{The Dreyfus-Wagner (DW) algorithm}

The algorithm is motivated by the fact that any SMT for instance $\mathcal{I}$ is guaranteed to consist of so-called sub-SMTs 8 . Given a vertex $u \in V$ and a set $I \subseteq R$, we define a sub-SMT $S$ for $(u, I)$ as an SMT for instance $(N, I \cup\{u\})$, i.e., $S \in \operatorname{smt}((N, I \cup\{u\}))$. Further, we denote by $l^{*}(u, I)$ the sub-SMT costs, $l^{*}(u, I)=\operatorname{csmt}(N, I \cup\{u\})$. The sub-SMTs of an SMT are vertex-disjoint apart from their corresponding roots. In other words, any SMT consists of sub-SMTs that are "joined" using their root vertices, which are referred to by join vertices. Then, intuitively, it suffices $[8]$ to incrementally compute sub-SMTs for parts of the instance and join them accordingly. Thereby, we create larger sub-SMTs, until finally ending at an SMT for $(N, R)$.

Example 3. Recall instance $\mathcal{I}=(N, R)$, where $R=\{a, b, c, d\}$ of Example 2 . In Figure 1 (right), vertex $F$ is a join vertex for the Steiner tree and vertex $F$ joins the sub-SMTs for instances $(N,\{a, b, F\}),(N,\{c, F\})$, and $(N,\{d, F\})$.

Listing 1 shows DW. The sub-SMT costs are computed for $(u, I)$ with increasing cardinalities of sets $I$. Sub-SMTs for $(u,\{v\})$ (singleton of ter-

minals) are computed using the distance between $u$ and $v$, cf., Line 2 . The 


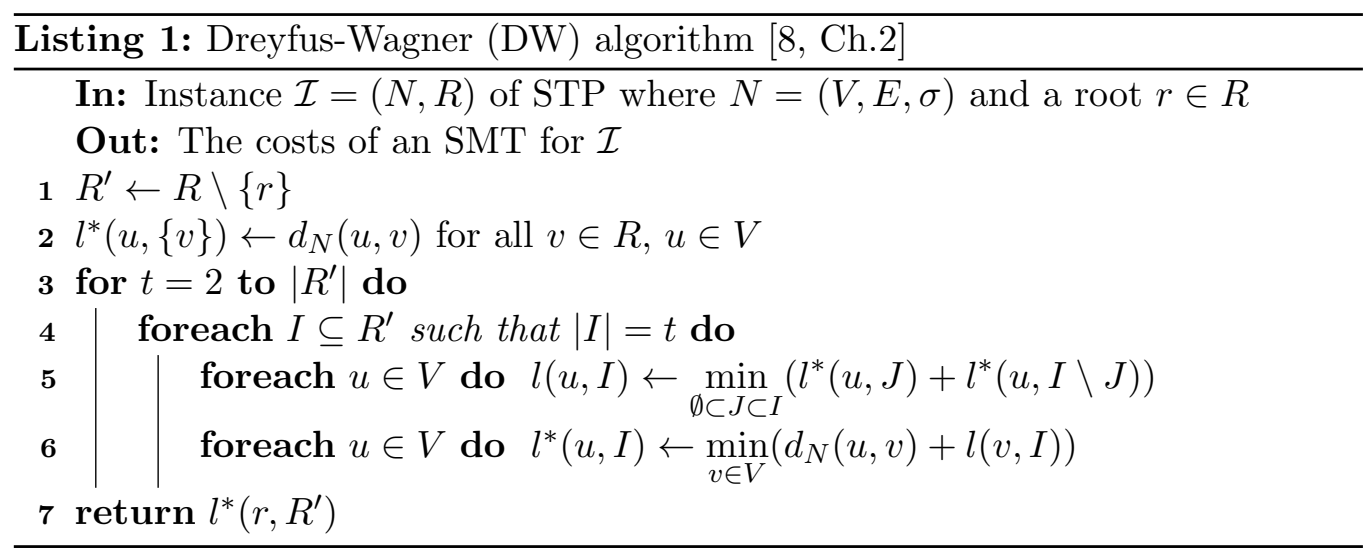

remaining sub-SMTs are computed in two steps. First, in step (i), a tentative cost value $l(u, I)$ is computed for every vertex $u \in V$ and each set $I$ of cardinality $t$, by combining costs $l^{*}(u, J)$ of sub-SMTs for $J \subsetneq I$ accordingly, cf., Line 5. Intuitively, this corresponds to joining two sub-SMTs at root $u$. Then, in Step (ii), cf., Line 6, sub-SMT costs $l^{*}(u, I)$ are computed for each vertex $u \in V$, by propagating costs $l(v, I)$ to all vertices $u \in V$. Intuitively, Line 6 corresponds to connecting a vertex $u$ to the sub-SMT for $(v, I)$ by a path between $u$ and $v$. After the algorithm terminates, $l^{*}(r, R \backslash\{r\})=\operatorname{csmt}(N, R)$. The SMT can then be found by retracing the steps of the algorithm.

Proposition $4([8,20])$. Given an instance $\mathcal{I}=(N, R)$ of $S T P$, where $N=$ $(V, E, \sigma)$. The $D W$ algorithm runs in time $\mathcal{O}\left(3^{|R|}\right)$ and space $\mathcal{O}\left(2^{|R|}|E|\right)$.

\subsection{The Dijkstra-Steiner (DS) algorithm}

In theory, the runtime of DW seems suitable for instances with a small number of terminals. In practice this algorithm consumes too much time and memory, even for about a few dozen of terminals. However, one can still lower the runtime by changing how the whole search space is explored. To this end, we first define for given instance $\mathcal{I}=(N, R)$ of STP the Steiner search network $\mathcal{N}(\mathcal{I}):=\left(V^{\prime}, E^{\prime}, \sigma^{\prime}\right)$, where the vertices are a set of pairs among $V \times 2^{R}$, i.e., $V^{\prime}:=\{(u, I), u \in V, \emptyset \subsetneq I \subseteq R \backslash\{r\}\}$. Then, there is an edge $e=\{(u, I),(v, J)\}$ in $E^{\prime}$ between any two distinct vertices $(u, I)$ and $(v, J)$, if we have either (1) $\{u, v\} \in \delta_{(V, E)}(u)$ and $I=J$; or $(2) u=v$ and $\emptyset \subsetneq J \subsetneq I$. The cost for each edge $e \in E^{\prime}$ are given as follows $\sigma^{\prime}(e):=w$, where $w=\sigma(\{u, v\})$ in Case $(1)$, and $w=l^{*}(v, I \backslash J)$ in Case (2). Since $\mathcal{N}(\mathcal{I})$ 
is a network, we can apply Dijkstra's algorithm to $\mathcal{N}(\mathcal{I})$. Since we cannot construct $\mathcal{N}(\mathcal{I})$ as $l^{*}$ is not known in advance, the algorithm runs on a partial network that is dynamically amended. During expansion of a vertex (in Dijkstra's algorithm), we consider only those neighboring tuples that are either adjacent in the network according to Case (1) or where $(u, I \backslash J)$ has been expanded before for Case (2). The improved algorithm is called the DijkstraSteiner (DS) algorithm [20]. DS has a worst-case runtime that is similar to DW. But DS does not expand vertices $(u, I)$, where $\operatorname{costs} l^{*}(u, I)>\operatorname{csmt}(N, R)$, which can cut down the runtime considerably in practice.

Another crucial tool to reduce the runtime is a guiding heuristic function and pruning the search space. A Steiner (guiding) heuristic function $h^{*}$ : $V \times 2^{R} \rightarrow \mathbb{N}$, provides a (cost) lower bound $\left.\right|^{2}$ on $l^{*}(u, I)$. Similar to the $\mathrm{A}^{*}$ algorithm, DS chooses in each iteration the tuple $(u, I)$ that minimizes $l^{*}(u, I)+h^{*}(u, R \backslash I)$. Thereby, the algorithm ignores tuples $(u, I)$ with $l^{*}(u, I)+h^{*}(u, R \backslash I)>\operatorname{csmt}(N, R)$, which can further reduce the number of expanded tuples. In addition to a heuristic function, DS uses pruning to speed up the search. An upper bound on costs is used to ignore tuples that do not contribute to an SMT. Given such a (cost) upper bound on any SMT for $(N, I)$, where $I \subseteq R$, we can ignore tuples $(u, I)$, where $l^{*}(u, I)$ exceeds this bound. Intuitively, this cuts down the number of considered tuples. Pruning and the Steiner heuristic function add their respective runtimes. While this increases the worst-case, it significantly reduces the runtime for most instances.

Listing 2 presents $D S^{*}$, the modified variant of DS. The modifications will be the topic of the next section. Since $l^{*}$ is not known in advance (as mentioned above), $\mathrm{DS}^{*}$ maintains tentative costs $l(u, I)$ for every tuple $(u, I)$, and uses set $P$ to track expanded tuples $(u, I)$. Note that, after termination of $\mathrm{DS}^{*}$, tentative costs $l(r, R \backslash\{r\})$ for root vertex $r$ are guaranteed to be the optimal costs of any SMT for the instance, i.e., $l(r, R \backslash\{r\})=l^{*}(r, R \backslash$ $\{r\})=\operatorname{csmt}(N, R)$. Further, DS keeps track of tuples $(u, I)$ contributing to $l^{*}(r, R \backslash\{r\})$, which is maintained in retrace set $b(r, R \backslash\{r\})$. In the end, retrace sets are used for retracing a (corresponding) SMT for $(N, R)$.

$\mathrm{DS}^{*}$ as presented in Listing 2 works similarly to DW. In particular, the two main steps of DW, (i) combining costs (Line 5) and (ii) propagating costs (Line 6), are performed in the loops in Line 12 and Line 17, respectively. However, the main difference between DW and $\mathrm{DS}^{*}$ is the sequence of opera-

\footnotetext{
${ }^{2}$ We provide a formal definition in the next subsection.
} 
tions. Namely, in contrast to DW, DS* does not process sets of terminals in order of increasing cardinality, but ordered by increasing estimated costs, as computed in Line 9. Unique to DS* is the pruning in Lines 16 and 21. Finally, after expansion of $\left(r, R^{\prime}\right)$, retraceSMT is used to combine sub-SMTs in order to construct an SMT. For this construction, we use retracing sets as collected in Lines 5, 15, and 20.

Example 5. Consider again our instance $\mathcal{I}=(N, R)$, where $N=(V, E, \sigma)$ from Example 2, and let $r:=c$ be the root node. In the following, we assume a perfect heuristic $h^{*}$, where $h^{*}(u, I)=\operatorname{csmt}(N, I \cup\{u\})$ for any vertex $(u, I)$ of $\mathcal{N}(\mathcal{I})$. Then, algorithm $D S^{*}$ requires 10 iterations. The necessary operations can be tracked using the SMT in Figure 1 (right), First, the initial tuples $(a,\{a\}),(b,\{b\})$ and $(d,\{d\})$, all with costs 0 , are expanded, cf., Line 10 of Listing 2. Next, the tuples $(b,\{a\})$ of cost 1 and $(I,\{d\})$ of cost 5 are expanded. Then, tuple $(b,\{a, b\})$ of cost 1 gets expanded. After expanding $(F,\{d\})$ of cost 10 and $(F,\{a, b\})$ cost 6 , we have the tuples needed for the set $R \backslash\{c\}$ of terminals. It remains to connect it to the root, which is achieved by expanding $(F,\{a, b, d\})$ of cost 16 and finally $(c,\{a, b, d\})$ of cost 22 yielding $\operatorname{csmt}(N,\{a, b, c, d\})=22$. This run creates 20 tuples in $Q$. Without the heuristic, i.e., assuming $h^{*}(u, I)=0$ for any vertex $(u, I)$ of $\mathcal{N}(\mathcal{I})$, every tuple $(v, J)$ with costs smaller than 22 has to be expanded. This takes about 55 iterations and 90 entries in $Q$. Note that this is still considerably less than the absolute worst case of about 160 iterations as in Listing $1(D W)$.

\subsection{Extended Dijkstra-Steiner $\mathrm{DS}^{*}$ for admissibility}

In this section, we discuss our changes to DS. Since $\mathcal{N}(\mathcal{I})$ is a dynamic graph structure and DS contains adaptions to the $\mathrm{A}^{*}$ algorithm correctness is not immediately obvious [20]. We therefore also discuss correctness of our new algorithm DS*

We establish the following definition to lift the existing correctness result of DS for any Steiner heuristic function that provides a cost lower bound.

Definition 6. Given an instance $\mathcal{I}=(N, R)$ of $S T P$, where $N=(V, E, \sigma)$ and $r \in R$, and a Steiner heuristic function $h^{*}: V \times 2^{R} \rightarrow \mathbb{N}$. Then, we say $h^{*}$ is

- admissible, if $h^{*}(u, I) \leq l^{*}(u, I)$ for every $u \in V$ and $\{r\} \subseteq I \subseteq R$; and 
- consistent, cf. [20], if $h^{*}(u, I) \leq h^{*}\left(v, I^{\prime}\right)+l^{*}\left(v,\left(I \backslash I^{\prime}\right) \cup\{u\}\right)$ for every $u, v \in V$ and $\{r\} \subseteq I^{\prime} \subseteq I \subseteq R$.

Intuitively, with this definition we lift the concept of admissibility of heuristic functions $h$ to Steiner heuristic functions $h^{*}$ and establish the perspective of admissibility and consistency in the context of Steiner heuristic functions. Similar to heuristic functions for $A^{*}$, an admissible Steiner heuristic function $h^{*}$ does not "over-approximate", i.e., it provides a lower bound, which is a generalization of the stricter consistency notion [20] that was used before. Note, that a vertex of the Steiner search network intuitively refers to several sub-graphs of potential Steiner trees and not to a simple path as used in $A^{*}$. As a result, a Steiner heuristic function goes beyond plain heuristic functions on top of the Steiner network. Next, we show that consistency still implies admissibility.

Proposition 7. Given an instance $(N, R)$ of $S T P$, where $r \in R$ and a consistent Steiner heuristic function $h^{*}$. Then, $h^{*}$ is admissible.

Proof (Sketch). We show that $h^{*}(u, I) \leq l^{*}(u, I)$ for every $u \in V,\{r\} \subseteq I \subseteq$ $R$, by induction on the cardinality of $I$. The base case of $|I|=|\{r\}|=1$ is similar to finding the shortest path in $N$ from $u$ to $r$, as used in $\mathrm{A}^{*}$. In particular, in this case consistency and admissibility of Steiner heuristic functions are a special case of consistency and admissibility of heuristic functions, respectively. Therefore, the result follows by the same argument [5], as in the original work for $\mathrm{A}^{*}$. In the induction step we show that $h^{*}(u, I) \leq$ $l^{*}(u, I)$ for every $u \in V,\{r\} \subseteq I \subseteq R$, assuming that $h^{*}(v, J) \leq l^{*}(v, J)$ for every $v \in V,\{r\} \subseteq J \subsetneq I$. Thereby, we use a result [8], which shows that for each vertex $u \in V$, either one of the cases holds:

1. $l^{*}(u, I)=l^{*}(u, J)+l^{*}(u, I \backslash J)$, with $J \subsetneq I$ : By consistency of $h^{*}$ and by the induction hypothesis, we have $h^{*}(u, I) \leq h^{*}(u, J)+l^{*}(u,(I \backslash$ $J) \cup\{u\}) \leq l^{*}(u, J)+l^{*}(u, I \backslash J) \leq l^{*}(u, I)$.

2. $l^{*}(u, I)=l^{*}(v, I)+d_{N}(u, v)$, for $u \neq v$, and for $v$ we have Case 1 . Then, we conclude by consistency of $h^{*}$ and by Case 1: $h^{*}(u, I) \leq$ $h^{*}(v, I)+l^{*}(v,(I \backslash I) \cup\{u\}) \leq l^{*}(v, I)+d_{N}(u, v)=l^{*}(u, I)$.

However, consistency is indeed strictly stronger, i.e., one can easily construct examples of non-consistent, admissible Steiner heuristic functions. 
Example 8. Consider network $N$ given in Figure 1 (left). Let $r:=c$ and $h^{*}$ be an admissible Steiner heuristic function. Take $h^{*}(d,\{c\}):=16$ and $h^{*}(F,\{c\}):=1$. Observe that $h^{*}$ is admissible, but not consistent, as $h^{*}(d,\{d\})>h^{*}(F,\{c\})+l^{*}(F,\{d\})$, i.e., $16>1+10$.

So far, correctness of DS is known for consistent Steiner heuristic functions [20]. In this case, consistency guarantees optimality of expanded tuples, i.e., for every expanded tuple $(u, I)$ (in $P$, cf., Listing 2), we have $l(u, I)=l^{*}(u, I)$. On the contrary, in case of mere admissibility, which, intuitively, ensures only a cost lower bound, the algorithm might find a lower value for $l(u, I)$ even after expansion of $(u, I)$, i.e. it only holds that $l(u, I) \geq l^{*}(u, I)$. Recall Listing 2, which indeed presents the $\mathrm{DS}^{*}$ algorithm. Actually, only Lines 18 and 13 of Listing 2 differ slightly from the original DS algorithm [20]. During expansion, $\mathrm{DS}^{*}$ explicitly considers already expanded tuples in $P$ since the tentative cost value $l(v, I)$ for an expanded tuple $(v, I) \in P$ is not guaranteed to be optimal.

Although the changes are small, the effect is significant: new Steiner heuristic functions can be used with $\mathrm{DS}^{*}$ and since the proof of correctness for DS strongly depends on tentative costs of expanded tuples being optimal, we have to use a different approach for showing correctness of $\mathrm{DS}^{*}$.

Theorem 9 ( $\star^{3}$, main result). For an instance $(N, R)$ of $S T P$ where $N=$ $(V, E, \sigma)$ and a root $r \in R$, the $D S^{*}$ Algorithm given in Listing 2, terminates if $h^{*}$ is admissible. Further, after termination, $l(r, R \backslash\{r\})$ is the cost of an SMT for the instance $(N, R)$, i.e., $l(r, R \backslash\{r\})=l^{*}(r, R \backslash\{r\}=\operatorname{csmt}(N, R)$.

Proof (Idea). Since $h^{*}$ is admissible, we have $h^{*}(r, R \backslash\{r\})=0$. By construction of DS* and admissibility of $h^{*}$, every tuple $(u, I)$ such that $l(u, I)+$ $h^{*}(u, R \backslash I)<l(r, R \backslash\{r\})$ is expanded. As a result, after termination, every tuple $(u, I)$ that "contributes" to $\operatorname{csmt}(N, R)$, is expanded beforehand.

While for consistent Steiner heuristic functions $h^{*}$, one obtains for DS a similar runtime result as for DW, cf., Proposition 4, this is not guaranteed for admissible functions $h^{*}$ and $\mathrm{DS}^{*}$. Intuitively, as described above, for non-consistent Steiner heuristic functions $h^{*}$, paths to arbitrary tuples $(v, I)$ are not necessarily cost optimal.

\footnotetext{
${ }^{3}$ Proofs of theorems marked with " $\star$ " can be found in the appendix.
} 
Theorem 10. Given an instance $\mathcal{I}=(N, R)$ of $S T P$, where $N=(V, E, \sigma)$, any root $r \in R$, and an admissible Steiner heuristic function $h^{*}$. Then, the $D S^{*}$ algorithm runs in time $\mathcal{O}\left(|R| \cdot\left(|V|+2^{|R|}\right)^{|V|+|R|}\right)$.

Proof (Sketch). Each vertex of the form $(u, I)$ in the Steiner network $\mathcal{N}(\mathcal{I})$ has by construction at most $|V|+2^{|R|-|I|}$ neighboring tuples in the network. Observe that an SMT of $\mathcal{I}$ consists of at most $|V|$ many vertices and has at most $|R|$ join vertices. Then, the length of a path from any tuple $(u, I)$ to $(r, R \backslash\{r\})$ in $\mathcal{N}$ that is discovered in $\mathrm{DS}^{*}$ is at most $|V|+|R|$, since a longer path would exceed $\operatorname{csmt}(N, R)$, causing algorithm $\mathrm{DS}^{*}$ to not expand the corresponding tuple. We therefore reach the goal $(r, R \backslash\{r\})$ after expanding at most $\left(|V|+2^{|R|-1}\right)^{|V|+|R|}$ vertices. In the worst case this is done for each terminal, except the root $r$. Hence, the bound holds.

In theory, the worst-case runtime may worsen significantly in case of mere admissibility, similar to the situation of exponential blow up for the $A^{*}$ algorithm [41]. However, in practice the ability to use non-consistent, but admissible heuristics often provides tighter bounds leading to shorter runtimes. The same is true for the $\mathrm{A}^{*}$ algorithm, where at first nobody could imagine merely admissible heuristic functions to outperform consistent ones [27, 41]. For $\mathrm{DS}^{*}$ as well as $\mathrm{A}^{*}$ it turns out that ILP based heuristic functions are both not consistent and often well-suited. In the next section, we discuss the Steiner heuristic functions used in our solver $\mathrm{DS}^{*}$ Solve.

\section{Implementation of $\mathrm{DS}^{*}$ : DS Solve}

We created the solver $D S^{*}$ Solve, a fully functional STP solver written in $\mathrm{C}++^{4}$. DS* Solve consists of two modules: the solving module, using $\mathrm{DS}^{*}$ as presented in Listing 2, and the preprocessing module, trying to reduce the complexity of the instance, before solving is started. In the following, we discuss implementation details regarding both these modules. Within this section, we again assume a given instance $(N, R)$, where $N=(V, E, \sigma)$ and a root $r \in V$.

\footnotetext{
${ }^{4} \mathrm{DS}^{*}$ Solve is publicly available at https://github.com/ASchidler/steiner_cpp 36.
} 


\subsection{Solving Module}

$\mathrm{DS}^{*}$ is implemented as previously described with the same pruning technique as originally suggested for DS [20]. The main improvement is the choice of Steiner heuristic functions, which is crucial for good results, as our experimental results show. We use two different Steiner heuristic functions and will discuss them subsequently: dual ascent and 1-tree. Given a lower bound $l b(N, I)$ of $\operatorname{csmt}(N, I)$ for any $I \subseteq R$, we define the corresponding Steiner heuristic function $h_{l b}^{*}$ (using $\left.l b\right)$ by $h_{l b}^{*}(u, I):=l b(N, I \cup\{u\})$ for any $u \in V, I \subseteq R$.

Dual Ascent [40, Section 3]. This admissible, but not consistent, Steiner heuristic function can only be used with $\mathrm{DS}^{*}$ and not DS. The idea is to use a feasible solution for the dual of the ILP formulation as a lower bound. The algorithm starts with edge costs $\sigma^{\prime}=\sigma$ and $|R \backslash\{r\}|$ sub-graphs of $(V, E)$, where each sub-graph $C_{t}$ consists of exactly one non-root terminal $t \in R \backslash\{r\}$. In each iteration one sub-graph $C_{t}$ is selected and extended. First, the set $E_{t}$ is computed: $E_{t}$ consists of all edges $e \in E$ incident to a node in $C_{t}$, but not in $C_{t}$. Next, the lowest cost $c^{\prime}=\min _{e \in E_{t}} \sigma^{\prime}(e)$ is determined. Then, all edges $e \in E_{t}$ with $\sigma^{\prime}(e)=c^{\prime}$ are added to $C_{t}$ and the costs of all edges in $E_{t}$ are reduced by $c^{\prime}$ in $\sigma^{\prime}$. Eventually, the sub-graphs will form one connected sub-graph $C_{r}$, containing all terminals including the root. Therefore, $C_{r}$ is a Steiner tree for the instance. The sum of all $c^{\prime}$ when adding an edge is a lower bound for $\operatorname{csmt}(N, R)$. Whenever we select a sub-graph $C_{t}$, we choose the sub-graph that has the minimal number of incident edges [31, 33. The algorithm runs in $\mathcal{O}(|E| \cdot \min \{|V| \cdot|R|,|E|\})|9|$.

1-Tree. Alternatively, DS* Solve uses the 1-Tree lower bound, whose corresponding Steiner heuristic function is consistent. This method is used in the original DS implementation [20].

Proposition 11 (1-tree lower bound). [20, Lemma 8] Given an STP instance $(N, R)$. Let $r \in R$ be any terminal and $R^{\prime}=R \backslash\{r\}$. Further, let $c$ be the cost of any minimum spanning tree of $D_{N}\left(R^{\prime}\right)$. Then, $\frac{1}{2}\left(c+\min _{u, v \in R^{\prime}: u \neq v \text { or }\left|R^{\prime}\right|=1}\left(d_{N}(r, u)+d_{N}(r, v)\right)\right.$ is a cost lower bound of any SMT of $(N, R)$.

The distance network can be constructed in time $\mathcal{O}(|V| \log (|V|)+|E|)$ and the minimum spanning tree for this network in time $\mathcal{O}\left(|R|^{2}\right)$. The lower bound can then be obtained in time $\mathcal{O}(|R|)[20]$.

$\mathrm{DS}^{*}$ Solve only uses one of the corresponding Steiner tree heuristic functions per instance. Oftentimes, dual ascent computes tighter bounds and DS* 
therefore requires fewer iterations to succeed. Unfortunately, the runtime for dual ascent also increases faster with increasing graph size than the runtime of 1-tree. Hence, we use dual ascent for graphs of up to 10.000 edges and use the 1-tree heuristic function for larger graphs.

The root terminal is not chosen arbitrarily. DS* Solve tries different terminals as the root with dual ascent. The terminal giving the highest lower bound is then used as the root. This strategy delivered superior results, compared to selecting the last terminal as used originally [20].

\subsection{Preprocessing Module}

Preprocessing for STP identifies and removes vertices and edges that are not required for an SMT. These methods usually run in time $\mathcal{O}(|V| \log (|V|))$ and can quickly reduce the complexity of an instance. Many instances would be too hard for the solving module alone, without preprocessing.

We use the standard methods described in literature [10,21]. Preprocessing is applied until no more reductions are possible. The order of preprocessing operations is chosen in a way to maximize reuse of calculated information, e.g. distances in the network. One noteworthy preprocessing method is based on the previously discussed dual ascent lower bound.

Dual ascent calculates a lower bound for $\operatorname{csmt}(N, R)$ and can be extended to provide a lower bound on the costs of any SMT containing a specific vertex or edge. Given an upper bound for $\operatorname{csmt}(N, R)$ we can remove any vertices and edges, where this lower bound exceeds the upper bound. We use different methods to obtain good upper bounds and thereby maximize the number of removals.

Upper bounds are obtained using the repeated shortest path heuristic (RSPH). RSPH is a well-established STP heuristic [4,37], that can compute Steiner trees in time $\mathcal{O}\left(|R| \cdot|V|^{2}\right)$. We can often find a tighter upper bound by running RSPH using only a sub-graph that contains all terminals. In addition to using the full instance, we run RSPH also using the following sub-graphs:

1. The sub-graph $C_{r}$ after a dual ascent run [33].

2. A preprocessed instance after guessing an upper bound 33.

3. A sub-graph obtained from combining several Steiner trees [33, 35].

Given a Steiner tree, we can also find a Steiner tree of lower costs by applying local search. This is done by systematically replacing sub-graphs of 
the tree in an effort to find a tree of smaller size. These sub-graphs may be single vertices or whole paths 38 .

\section{Experimental Work}

We conducted a series of experiments using standard benchmark instances for STP. Instances and result: 5 , including raw data, are publicly available. Our experimental work aims for a comparison between different heuristics in order to understand whether our proposed extensions are valuable in practice. Further, we compare the effectiveness of our prototypical solver with other established implementations.

Benchmark Instances. We considered a selection of overall 1.623 instances, which originate from the 11th DIMACS Challenge [24] and PACE 2018 Challenge [2]. We group them as done in the literature [23].

1. SteinLib, which contain generated graphs with random costs (random), artificial instances (artificial), instances with euclidean weights (euclidean), cross-grid graphs (crossgrid), grid graphs with holes (vlsi), randomly generated rectilinear instances (rectilinear), wire routing problem based instances (group);

2. $\operatorname{Cph} 14$

(simplified obstacle-avoiding rectilinear instances);

3. Vienna

(real world instances from telecommunication networks);

4. PACE2018 (PACE 2018 challenge instances).

Measure, Setup, and Resource Enforcement. Our results were gathered on a cluster running Ubuntu 18.04.3 LTS (kernel 4.15.0-101-generic) and GCC 7.5.0. Each node is equipped with two Intel Xeon E5-2640v4 CPUs and 160GB RAM. We limited the solvers to 1800 seconds wall clock time and 8GB of RAM per instance. We used reprobench to setup the benchmarks and to enforce the resource limits.

\footnotetext{
${ }^{5}$ See: 12 .

${ }^{6}$ https://github.com/rkkautsar/reprobench
} 
Benchmarked Solvers. We tested three configurations of our solver: DS*, which implements our enhanced algorithm without preprocessing, DS* Solve, which in addition includes preprocessing, and $-d a$, which on uses the 1-tree heuristic function. We test the solver $H S V^{7}$, which is known as a successful implementation of DS algorithm [20]. We include the best PACE 2018 solvers Pruned ${ }^{8}$ (2700bdc/Rust1.36.0) 23] and SCIP-Jack $\left.\right|^{9}$ (6.0.2/SoPlex4.0.2).

Experiment 1. In order to benchmark the effectiveness of our enhanced algorithm and a comparison to plain DS, we take $\mathrm{DS}^{*}$ and the solver $H S V$ into account. Since one might argue that also implementation specific tricks and algorithm engineering might have a strong influence on the number of solved instances, we also tested our solver in the configuration $-d a$, which disables the dual ascent heuristic and assembles a solver that is close to the underlying algorithm of $H S V$. Since $H S V$ can handle only instances of less than 64 terminals, we restrict the instances accordingly.

Result: Table 1 the results on the number of solved instances. DS* without dual ascent performs worse than HSV. DS* performs better on all sets. If we subgroup SteinerLib, one can observe that it particularly helps to solve randomly generated graphs (random) and wire routing problem based instances (group).

Discussion: Besides implementation details, DS*-da performs worse than $H S V$. We suspect that the main reason is the use of the Steiner heuristic functions in $H S V$, which is slightly more sophisticated than the 1-tree heuristic function [20]. The results show that using merely admissible Steiner heuristic functions can significantly improve the number of solved instances.

Experiment 2. In order to contrast our algorithm and implementation with approaches and their implementations, we take various state-of-the-art solvers in the field into account.

Results: In Table 2, we report the number of solved instances for the implemented techniques. Depending on the instance set, we observe a varying number of solved instances. Overall, SCIP solves the most instances. DS* Solve solves 237 instances less and Pruned another 39 instances less. If we subgroup SteinLib, DS*Solve and Pruned solve more instances than SCIP on vlsi. Pruned solves more instances than SCIP on group. SCIP solves almost 40 and 80 instances more on the sets rectlinear and random, respec-

\footnotetext{
${ }^{7}$ We thank the authors for providing us with a copy of the solver.

8 https://github.com/wata-orz/steiner_tree

9 https://scip.zib.de/
} 


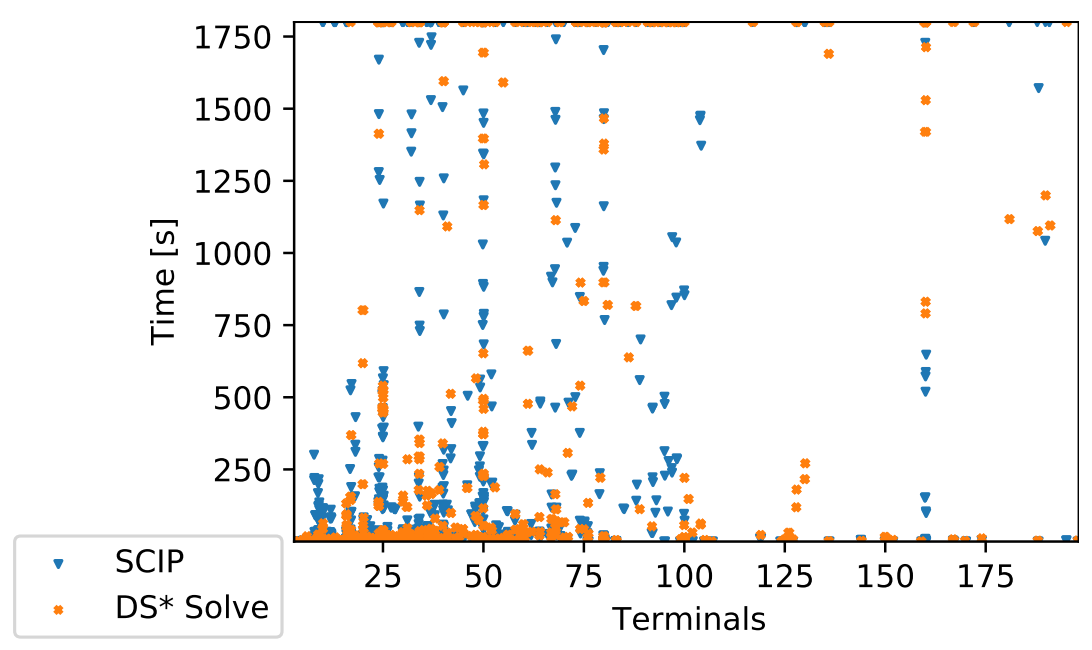

Figure 2: Runtime of $\mathrm{DS}^{*}$ Solve vs $S C I P$. The x-axis labels the number of terminals of the instance and $y$-axis captures the runtime in seconds.

tively, and similar many or a few more on the remaining sets. Figure 2 shows a runtime comparison between DS* Solve and SCIP based on the instance's number of terminals. We observe that for lower numbers DS* Solve is usually faster than SCIP.

Discussion: Similar as in the previous experiment, we can see that there is a big difference between using consistency and admissibility, namely 152 solved instances. If we compare DS* Solve to Pruned, we observe that overall the former solves more instances. Since Pruned performs better on some groups of the set SteinLib sets, a combination of both approaches might produce a better overall solver. While $S C I P$ solves the most instances mainly due to the high number of solved instances in the sets rectlinear and random, DS* Solve solves instances with a low number of terminals very fast and often much faster than $S C I P$. Hence, we expect good performance as part of a portfolio solver.

\section{Conclusion and Future Work}

In this paper, we established the concept of admissible Steiner heuristic functions for the Steiner tree problem. We focused on instances with few terminals, and lifted the so-called Dijkstra-Steiner (DS) algorithm from consistent heuristic functions to admissibility, resulting in the DS* algorithm. 
Intuitively, admissibility of heuristic functions only requires a weak condition for lower bounds. More precisely, an admissible heuristic function is guaranteed to never over-approximate the actual costs, and is indeed strictly weaker than consistency. Admissible heuristic functions enable lower bound computation based on LP techniques, as for example the dual ascent method. Our solver DS* Solve combines the usage of admissible heuristic functions during solving, efficient preprocessing techniques, and methods for obtaining strong upper bounds. Experiments indicate that admissible heuristic functions have a strong effect on the solving performance.

An interesting question for future work is the integration of the dual ascent technique into other approaches and solvers. 


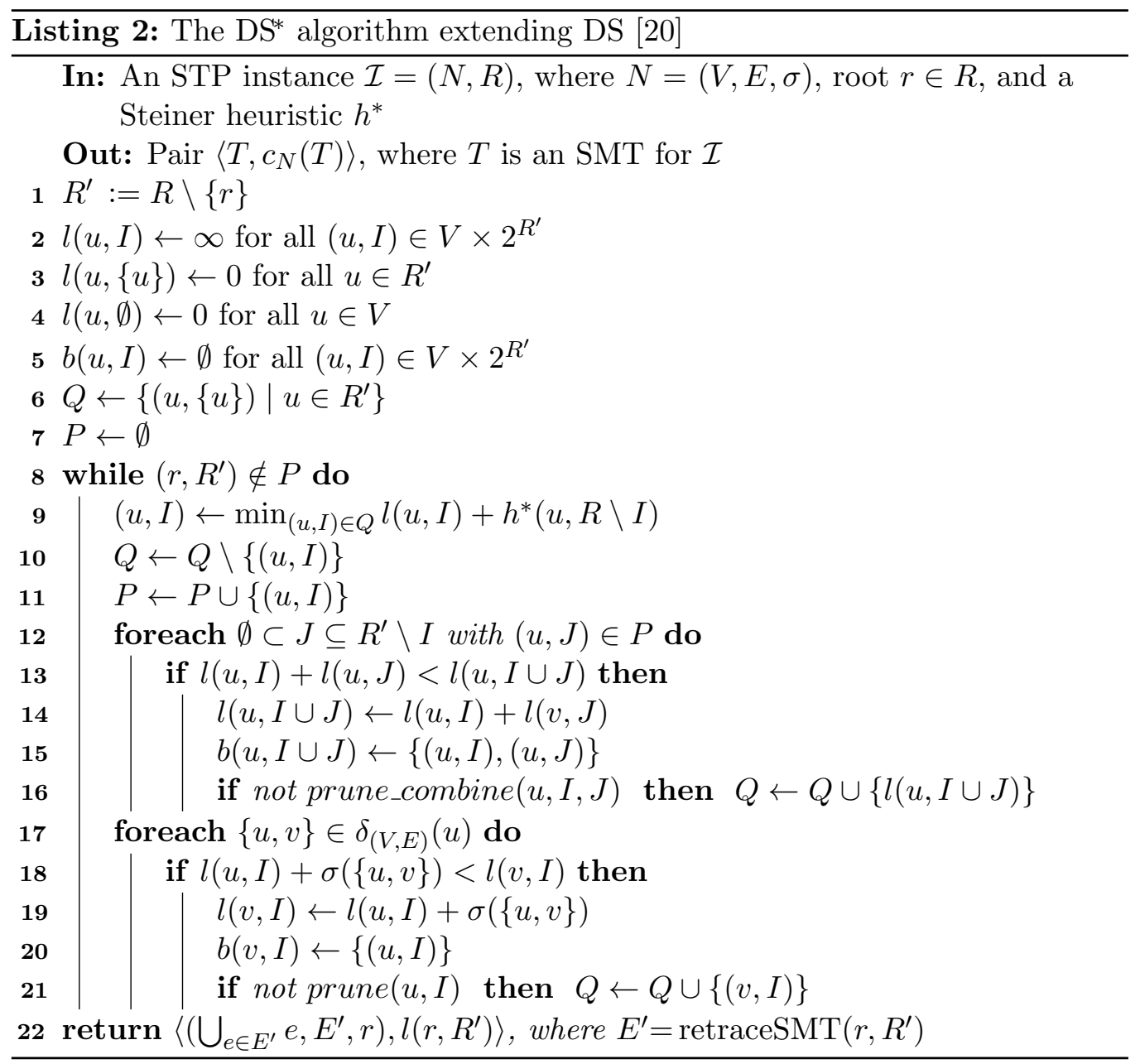

Given vertex $u \in V$ and $I \in 2^{R}$. Then, $\operatorname{retraceSMT}(u, I) \quad:=$ $\bigcup_{\left(u^{\prime}, I^{\prime}\right) \in b(u, I)} \operatorname{retraceSMT}\left(u^{\prime}, I^{\prime}\right) \cup\left\{\left\{u, u^{\prime}\right\} \mid u \neq u^{\prime}\right\}$. 


\begin{tabular}{lrrrr}
\hline Set & N & DS $^{*}$ & DS $^{*}$-da & $H S V$ \\
\hline SteinLib & 820 & $\mathbf{6 9 1}$ & 522 & 557 \\
Cph14 & 10 & $\mathbf{1 0}$ & $\mathbf{1 0}$ & $\mathbf{1 0}$ \\
Vienna & 6 & $\mathbf{4}$ & 3 & 3 \\
PACE2018 & 195 & $\mathbf{1 8 5}$ & 157 & 157 \\
\hline$\Sigma$ & 1031 & $\mathbf{8 9 0}$ & 692 & 727 \\
\hline
\end{tabular}

Table 1: Number of solved instances with less than 64 terminals for DS-based solvers. $\mathrm{DS}^{*}$-da lists the results for $\mathrm{DS}^{*}$ without dual ascent heuristic. $N$ indicates the total number of instances.

\begin{tabular}{lrrrrr}
\hline Set & $\mathrm{N}$ & DS $^{*}$ Solve & DS $^{*}$ Solve-da & Pruned & SCIP \\
\hline SteinLib & 1186 & 928 & 796 & 874 & $\mathbf{1 0 5 5}$ \\
Cph14 & 21 & 15 & 12 & 16 & $\mathbf{1 7}$ \\
Vienna & 216 & 11 & 5 & 26 & $\mathbf{1 2 0}$ \\
PACE2018 & 200 & $\mathbf{1 8 9}$ & 178 & $\mathbf{1 8 9}$ & 178 \\
\hline$\Sigma$ & 1623 & 1143 & 991 & 1105 & $\mathbf{1 3 7 0}$ \\
\hline
\end{tabular}

Table 2: Number of solved instances for the considered solvers. $N$ indicates the overall number of instances. 


\section{References}

[1] Hans L. Bodlaender, Marek Cygan, Stefan Kratsch, and Jesper Nederlof. Deterministic single exponential time algorithms for connectivity problems parameterized by treewidth. Information and Computation, 243:86-111, 2015.

[2] Édouard Bonnet and Florian Sikora. The PACE 2018 Parameterized Algorithms and Computational Experiments Challenge: The Third Iteration. In IPEC'18, volume 115 of LIPIcs, pages 26:1-26:15. Dagstuhl Publishing, 2019.

[3] Marek Cygan, Fedor V. Fomin, Łukasz Kowalik, Daniel Lokshtanov, Marcin Pilipczuk Dániel Marx, Michał Pilipczuk, and Saket Saurabh. Parameterized Algorithms. Springer, 2015.

[4] M. P. de Aragão and R. F. Werneck. On the Implementation of MSTBased Heuristics for the Steiner Problem in Graphs. In Algorithm Engineering and Experiments, pages 1-15. Springer, 2002.

[5] Rina Dechter and Judea Pearl. Generalized best-first search strategies and the optimality of a*. J. ACM, 32(3):505-536, July 1985.

[6] Reinhard Diestel. Graph Theory, 4th Edition, volume 173 of Graduate Texts in Mathematics. Springer Verlag, 2012.

[7] E. W. Dijkstra. A Note on Two Problems in Connexion with Graphs. Numerische Mathematik, 1:269-271, 1959.

[8] Stuart E. Dreyfus and Robert A. Wagner. The Steiner problem in graphs. Networks, 1:195-207, 1972.

[9] C. W. Duin. Steiner's Problem in Graphs. PhD thesis, University of Amsterdam, 1993.

[10] C. W. Duin and A. Volgenant. Reduction Tests for the Steiner Problem in Graphs. Networks, 19(5):549-567, 1989.

[11] R. E. Erickson, C. L. Monma, and A. F. Veinott. Send-and-Split Method for Minimum-Concave-Cost Network Flows. Mathematics of Operations Research, 12(4):634-664, 1987. 
[12] Johannes K. Fichte, Markus Hecher, and André Schidler. Raw data on experiments for DS Star, September 2020.

[13] Matteo Fischetti, Markus Leitner, Ivana Ljubić, Martin Luipersbeck, Michele Monaci, Max Resch, Domenico Salvagnin, and Markus Sinnl. Thinning out Steiner trees: A node-based model for uniform edge costs. Mathematical Programming Computation, 9(2):203-229, 2017.

[14] G. Gamrath, T. Koch, J. Maher, D. Rehfeldt, and Y. Shinano. SCIPJack - a solver for STP and variants with parallelization extensions. Mathematical Programming Computation, 9(2):231-296, 2017.

[15] Edgar N. Gilbert. A solvable routing problem. Networks, 19(5):587-594, 1989.

[16] Martin Grötschel, Alexander Martin, and Robert Weismantel. The steiner tree packing problem in vlsi design. Mathematical Programming, 78(2):265-281, Aug 1997.

[17] Dan Gusfield. Algorithms on Strings, Trees, and Sequences: Computer Science and Computational Biology. Cambridge University Press, Cambridge, 1997.

[18] Peter Hart, Nils Nilsson, and Bertram Raphael. A formal basis for the heuristic determination of minimum cost paths. IEEE Transactions of Systems Science and Cybernetics, 4(2):100-107, 1968.

[19] Mathias Hauptmann and Marek Karpinski. A compendium on Steiner tree problems. http://theory.cs.uni-bonn.de/info5/ steinerkompendium/, 2015.

[20] Stefan Hougardy, Jannik Silvanus, and Jens Vygen. Dijkstra meets Steiner: A fast exact goal-oriented Steiner tree algorithm. Mathematical Programming Computation, 9(2):135-202, 2016.

[21] Frank K. Hwang, Dana S. Richards, and Pawel Winter. The Steiner Tree Problem, volume 53 of Annals of Discrete Mathematics. Elsevier, 1992.

[22] Yoichi Iwata and Takuto Shigemura. wata_sigma: Steiner tree solver. https://github.com/wata-orz/steiner_tree, 2018. 
[23] Yoichi Iwata and Takuto Shigemura. Separator-based pruned dynamic programming for steiner tree. In AAAI 2019, volume 33, pages 1520-1527, 2019 .

[24] David S. Johnson, Thorsten Koch, Renato F. Werneck, and Martin Zachariasen. 11th dimacs implementation challenge in collaboration with icerm: Steiner tree problems. http://dimacs11.zib.de/, 2014.

[25] Richard M. Karp. Reducibility among combinatorial problems. In Complexity of computer computations, pages 85-103. Plenum, 1972.

[26] Thomas L. Magnanti and Richard T. Wong. Network design and transportation planning: Models and algorithms. Transportation Science, 18(1):1-55, 1984.

[27] A. Martelli. On the Complexity of Admissible Search Algorithms. Artificial Intelligence, 8:1-13, 1977.

[28] Krzysztof Maziarz and Adam Polak. Jagiellonian: Exact steiner tree with few terminals. https://bitbucket.org/krismaz/pace2018/src/ master/, 2018.

[29] Peter Mitura and Ondřej Suchý. Fit ctu: Steiner tree solver for pace 2018. https://github.com/PMitura/pace2018, 2018.

[30] Jesper Nederlof. Fast polynomial-space algorithms using inclusionexclusion: Improving on Steiner tree and related problems. Algorithmica, 65(4):868-884, 2013.

[31] Thomas Pajor, Eduardo Uchoa, and Renato F. Werneck. A robust and scalable algorithm for the Steiner problem in graphs. Mathematical Programming Computation, 10(1):69-118, 2018.

[32] Christos H. Papadimitriou. Computational Complexity. Addison-Wesley, 1994.

[33] T. Polzin. Algorithms for the Steiner Problem in Networks. PhD thesis, Universität des Saarlandes, 2003.

[34] Daniel Rehfeldt. A generic approach to solving the Steiner tree problem and variants. Master's thesis, Konrad-Zuse-Zentrum für Informationstechnik, 2015. 
[35] C. C. Ribeiro, E. Uchoa, and R. F. Werneck. A Hybrid Grasp with Pertubations and Adaptive Path-Relinking for the Steiner Problem in Graphs, 2000.

[36] André Schidler, Markus Hecher, and Johannes K. Fichte. DS Star - a Steiner tree solver, September 2020.

[37] H. Takahashi and A. Matsuyama. An approximate solution for the steiner problem in graphs. Mathematica Japonica, 24(6):573-577, 1980.

[38] E. Uchoa and R. F. Werneck. Fast Local Search for the Steiner Problem in Graphs. ACM Journal of Experimental Algorithmics, 17(2):2.2:1-2.2:222, 2012.

[39] Tom van der Zanden. SteinerTreeTW. https://github.com/ TomvdZanden/SteinerTreeTW, 2018.

[40] R. T. Wong. A Dual Ascent Approach for Steiner Tree Problems on a Directed Graph. Mathematical Programming, 28(3):271-287, 1984.

[41] Z. Zhang, N. R. Sturtevant, R. Holte, J. Schaeffer, and A. Felner. A* Search with Inconsistent Heuristics. In IJCAI 2009, pages 634-639, 2009. 


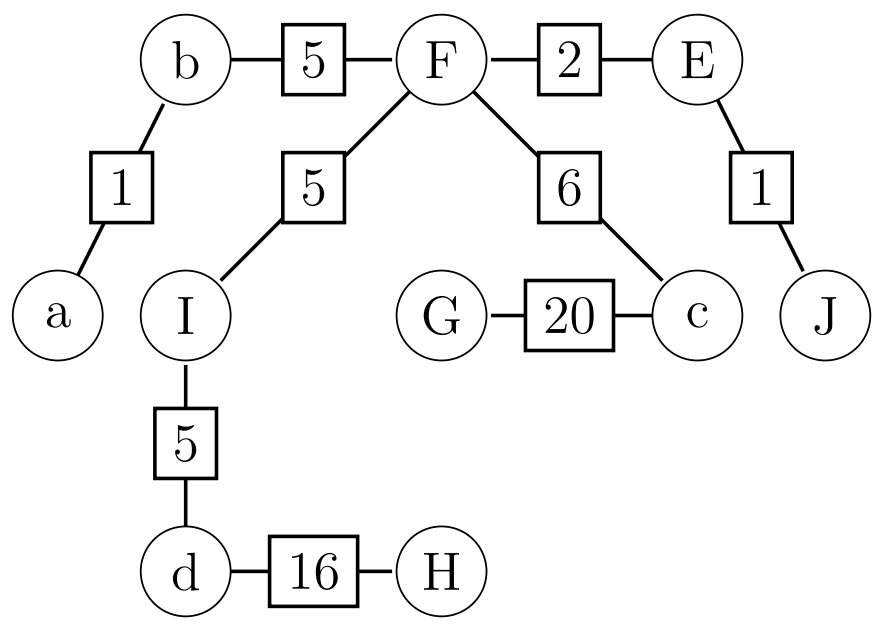

Figure 3: An MST of network $N$ in Figure 1

\section{A Additional Examples}

\section{A.1 MST of our example network.}

Example 12. Recall instance $\mathcal{I}=(N, R)$ from Example 2. Further, Figure 3 shows the MST for network $N$ of Figure 1 .

\section{A.2 Example of the DW algorithm.}

Example 13. Table 3 shows the result of a full $D W$ run for instance $\mathcal{I}=$ $(N, R)$, where $N=(V, E, \sigma)$ as given in Example 2, where $c$ is used as a root. The table shows vertically the vertices $v \in V$, whereas horizontally subsets $I \subseteq 2^{R \backslash\{c\}}$ are depicted. Depending on the column, the cells either show value $l(v, I)$ or $l^{*}(v, I)$, respectively. The subsets are ordered by increasing cardinality. As the computation is quite tedious, we focus on specific ones, namely on the row for the vertex $c$. The computation thereby proceeds column by column from left to right.

For subsets of cardinality one, values of $l^{*}(c,\{a\}), l^{*}(c,\{b\}), l^{*}(c,\{d\})$ are simply the distances between $c$ and $a, b$ and $d$, respectively. Therefore, the corresponding values in the table are 12, 11 and 16, respectively. For the value of $l(c,\{a, d\})$ we have to consider the values of all disjoint subsets that comprise $\{a, d\}$. There is only one such decomposition, $(\{a\},\{d\})$, and the value of $l(c,\{a, d\})$ is therefore $l^{*}(c,\{a\})+l^{*}(c,\{d\})=12+16=28$. 


\begin{tabular}{|c|c|c|c|c|c|c|c|c|c|c|c|}
\hline & $\{a\}$ & $\{b\}$ & $\{d\}$ & \multicolumn{2}{|c|}{$a, b$} & \multicolumn{2}{|c|}{$a, d$} & \multicolumn{2}{|c|}{$b, d$} & \multicolumn{2}{|c|}{$a, b, d$} \\
\hline Vertex & $l^{*}$ & $l^{*}$ & $l^{*}$ & $l$ & $l^{*}$ & & $l^{*}$ & $l$ & $l^{*}$ & $l$ & $l^{*}$ \\
\hline $\mathrm{a}$ & 0 & 1 & 16 & 1 & 1 & 16 & 16 & 17 & 16 & 16 & \\
\hline$b$ & 1 & 0 & 15 & 1 & 1 & 16 & 16 & 15 & 15 & 16 & \\
\hline $\mathrm{c}$ & 12 & 11 & 16 & 23 & 17 & 28 & 22 & 27 & 26 & 33 & 22 \\
\hline d & 16 & 15 & 0 & 31 & 16 & 16 & 16 & 15 & 15 & 31 & \\
\hline $\mathrm{E}$ & 8 & 7 & 12 & 15 & 8 & 20 & 18 & 19 & 17 & 20 & \\
\hline $\mathrm{F}$ & 6 & 5 & 10 & 11 & 6 & 16 & 16 & 15 & 15 & 16 & \\
\hline $\mathrm{G}$ & 32 & 31 & 36 & 63 & 32 & 68 & 42 & 67 & 41 & 68 & \\
\hline $\mathrm{H}$ & 32 & 31 & 16 & 63 & 32 & 48 & 42 & 47 & 41 & 48 & \\
\hline I & 11 & 10 & 5 & 21 & 11 & 16 & 16 & 15 & 15 & 16 & \\
\hline $\mathrm{J}$ & 9 & 8 & 13 & 17 & 9 & 22 & 19 & 21 & 18 & 22 & \\
\hline
\end{tabular}

Table 3: The full Dreyfus-Wagner computation for our running example.

For the computing values of $l^{*}$ for set $\{a, d\}$, we have to consider the values of $l$ for all other vertices and add the distance to $c$. We therefore compute $l(v,\{a, d\})+d_{N}(v, c)$ for all vertices $v \in V$. The corresponding values are $a: 16+12=28, b: 16+11=27, d: 16+16=32, E: 20+8=28$ and $F: 16+6=22$. The values in relation to any other vertex would be higher, so we ignore them. We keep the minimum of 22 as value for $l^{*}(c,\{a, d\})$.

Next, we compute the value of $l$ for $\{a, b, d\}$. We therefore have to consider the subset tuples $(\{a, b\},\{d\}),(\{a\},\{b, d\})$ and $(\{a, d\},\{b\})$. The respective values are $17+16=33,12+26=38$ and $22+11=33$. We again retain the minimum value 33 .

The final tuple we need, is the value of $l^{*}$ for $\{a, b, d\}$. We again use the $l$ values of all other vertices. We again need only vertices up to $F$ as $l(F,\{a, b, d\})+d_{N}(c, F)$ is the minimum value. The computations are $a: 16+12=28, b: 16+11=27, d: 31+16=47, E: 20+8=28$ and $F: 16+6=22$. We now have found our solution: $22=l^{*}(c,\{a, b, d\})$.

\section{B Further algorithmic details}

\section{B.1 Dual Ascent for STP.}

The algorithm is shown in Listing 3 and takes as argument an instance $\mathcal{I}=$ $(N, R)$ of STP, where $N=(V, E, \sigma)$, and $r \in R$. In the algorithm, $Q$ stores the active terminals, $\tilde{\sigma}$ the updated costs of (directed) edges, and $\tilde{w}$ keeps 


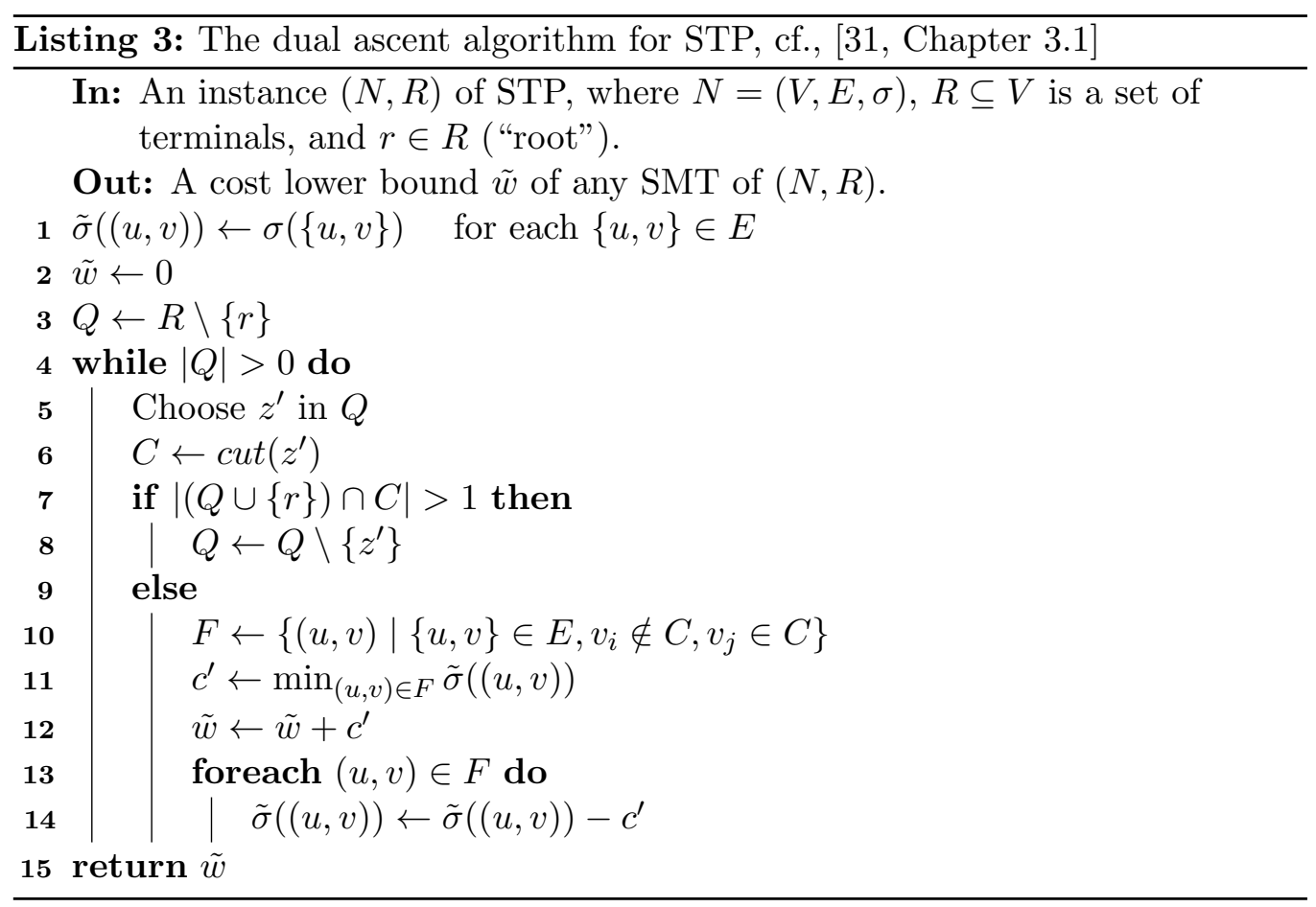

track of the lower bound. Function cut computes for a given terminal $z$ the corresponding subgraph by regarding every edge with 0 costs as part of the subgraph. The algorithm runs in time $\mathcal{O}(|E| \cdot \min \{|V| \cdot|R|,|E|\})[9]$.

\section{B.2 Pruning for DS.}

Next, we discuss the pruning strategy for a given instance $(N, R)$, where $N=$ $(V, E)$, and $r \in R$, as used in Lines 16 and 21 of Listing 2. We limit ourselves to the definition and refer the reader to the literature [20] for further details. Pruning works by comparing tentative $\operatorname{costs} l(v, J)$ for a tuple $(v, J)$ against an upper bound $U(J)$, which is maintained for pruning. Whenever $l(v, J)$ exceeds $U(J)$ the prune and prune_combine functions calls return true, since exceeding this upper bound implies that $(v, J)$ is not a sub-SMT for the instance and is therefore disregarded by the algorithm.

Whenever prune is called with arguments $v$ and $J$, it updates $U$. To this end, we define the set-distance $s d: 2^{V} \times 2^{V} \rightarrow \mathbb{N}$ as $s d(A, B):=$ $\min _{x \in A, y \in B} d_{N}(x, y)$. We then update $U(J) \leftarrow \min \{U(J), l(v, J)+\min \{s d(J, R \backslash$ $J), s d(\{v\}, R \backslash J\}\}$. Additionally, we maintain a set $S(J)$. Whenever prune 
changes $U(J)$, we have then exactly one $z \in R \backslash J$ that was the reason for finding the minimum value using $s d$ in $U(J)$. We then set $S(J) \leftarrow\{z\}$.

Function prune_combine is an extension of prune, that tries to combine upper bounds, before proceeding as described above. Whenever the method is called with arguments $J_{1}$ and $J_{2}$, it checks if $U\left(J_{1}\right)$ and $U\left(J_{2}\right)$ are defined. If this is the case and either $S\left(J_{1}\right) \cap J_{2}=\emptyset$ or $S\left(J_{2}\right) \cap J_{1}=\emptyset$, then the two upper bounds are combined. This is done by setting $U\left(J_{1} \cup J_{2}\right) \leftarrow U\left(J_{1}\right)+U\left(J_{2}\right)$ and $S\left(J_{1} \cup J_{2}\right) \leftarrow\left(S\left(J_{1}\right) \cup S\left(J_{2}\right)\right) \backslash\left(J_{1} \cup J_{2}\right)$.

\section{Correctness Proof of DS* for Admissibility}

In this section, we consider again an instance $\mathcal{I}=(N, R)$ of STP, where $N=$ $(V, E, \sigma)$, and $r \in R$. Further, let $h^{*}$ be an admissible, but not necessarily consistent Steiner heuristic function. We prove that algorithm $\mathrm{DS}^{*}$ as presented above in Listing 2 is still correct for $h^{*}$. To simplify the proof, we show this without pruning, i.e., we assume that there is no pruning and therefore the if-conditionals in Lines 16 and 21 of Listing 2 are always true. The proof of correctness including pruning can be established by showing that the invariants still hold. Intuitively, pruning uses tentative costs $l(u, I)$ of tuples $(u, I)$ to compute an upper bound, if the value is non-optimal, which then mainly increases practical efficiency by still preserving validity.

We reuse the following results from the correctness proof [20] of DS, that do not depend on the consistency of $h^{*}$.

R1 For each $(u, I) \in P \cup Q$, we have:

R1.1 $b(u, I) \subseteq P$ and retraceSMT $(u, I)$ returns the set of edges of a connected graph $T$ such that all vertices $\{u\} \cup I$ are in $T$ and $c_{N}(T) \leq l(u, I)$, and

$\mathrm{R} 1.2 I \cup\{u\}=\bigcup_{(v, J) \in b(u, I)} J \cup\{u\}$.

R2 For each $(u, I) \in\left(V \times 2^{[R \backslash\{r\}]}\right) \backslash P$, we have:

$\mathrm{R} 2.1 l(u, I) \geq \operatorname{csmt}(N,\{u\} \cup I)$, and

$\mathrm{R} 2.2$ if $l(u, I)=\operatorname{csmt}(N,\{u\} \cup I)$, then $(u, I) \in Q$.

We also introduce a few definitions. We denote by tree $(u, I)$ the graph defined by the set retraceSMT $(u, I)$ of edges. Furthermore we define a tuple $(u, I)$ 
to be optimal, if $l(u, I)=\operatorname{csmt}(N, I \cup\{u\})$ and tree $(u, I)$ is a subgraph of at least one $T \in \operatorname{smt}(\mathcal{I})$. Note that the tentative costs of an optimal tuple are never "overwritten", since any new tuple cannot have lower weight by $R 1.1$ and R2.1.

We define the transformation $\operatorname{tr}\left(u, T_{1}, T_{2}\right)$ for two trees $T_{1}=\left(V_{1}, E_{1}, \cdot\right), T_{2}=$ $\left(V_{2}, E_{2}, \cdot\right)$, where $T_{1}$ is a subgraph of $T_{2}$ and $u \in V_{1}$. Let $V^{\prime}=V_{2} \backslash\left(V_{1} \backslash\{u\}\right)$ and $E^{\prime}=\left(E_{2} \backslash E_{1}\right)$. The result of the transformation $\operatorname{tr}\left(u, T_{1}, T_{2}\right)$ is the graph $\left(V^{\prime}, E^{\prime}\right)$. Intuitively, the result of $t r$ is the graph obtained after removing from $T_{2}$ the edges in $T_{1}$.

Furthermore, we define $\operatorname{tr}_{v}\left(u, T_{1}, T_{2}\right)$ for trees $T_{1}=\left(V_{1}, E_{1}, \cdot\right), T_{2}=$ $\left(V_{2}, E_{2}, \cdot\right)$ s.t. $T_{1}$ is a subgraph of $T_{2}$ and $u \in V_{1}, r \in V_{2}$, as follows. Let therefore $T^{\prime}=\operatorname{tr}\left(u, T_{1}, T_{2}\right)$. Since $T^{\prime}$ is acyclic, there exists a unique path $S_{u, v}$ from $u$ to $v$. Let $T^{\prime \prime}=\operatorname{tr}\left(u, S_{u, v}, T^{\prime}\right)=\left(V^{\prime \prime}, E^{\prime \prime}\right)$ and $C=\left(V_{C}, E_{C}\right)$ be the largest subgraph of $T^{\prime \prime}$ containing $u$. The result of the transformation $\operatorname{tr}_{v}\left(u, T_{1}, T_{2}\right)$ is the graph $\left(V_{C}, E^{\prime \prime} \cap\left(V_{C} \times V_{C}\right)\right)$. After the transformation, only the connected subgraph containing $u$, without $T_{1}$, remains of $T_{2}$.

Lemma 14 (Loop Invariant). The following invariants hold at the beginning of every iteration of the main loop in Line 8 of Listing 2 . There exists a partition $\mathcal{W}$ of set $R \backslash\{r\}$ of vertices such that:

H1 There exists $T \in \operatorname{smt}(\mathcal{I})$, such that

H1.1 for each $W \in \mathcal{W}$, there exists $(u, W) \in P \cup Q$ such that tree $(u, W)$ is a subgraph of $T$ and $(u, W)$ is optimal, and

H1.2 for each $(u, W) \in P \cup Q$, such that tree $(u, W)$ is a subgraph of $T$, there exists no $W^{\prime} \in \mathcal{W}$ with $W \neq W^{\prime}$ such that $\left(u, W \cup W^{\prime}\right)$ is optimal, tree $\left(u, W^{\prime}\right)$ is a subgraph of $T$ and $\left(u, W \cup W^{\prime}\right) \in P \cup Q$.

H2 For each $W \in \mathcal{W}$ we have

H2.1 there exists a tuple $(u, W) \in Q$ such that $(u, W)$ is optimal, or

H2.2 there exists a tuple $(u, W) \in P$ such that $(u, W)$ is optimal. Furthermore, there exists $T \in \operatorname{smt}(\mathcal{I})$ such that tree $(u, W)$ is a subgraph of $T$ and $\left|R \cap V^{\prime}\right|>0$, where $\left(V^{\prime}, E^{\prime}\right)=\operatorname{tr}_{r}(u$, $\operatorname{tree}(u, W), T)$.

In the following, we refer to the invariant conditions by the corresponding label. Before we prove the lemma, we prove some results of this invariant. To this end, we let for given $T \in \operatorname{smt}(\mathcal{I})$ and tuple $(u, I), \phi(u):=R \cap V^{\prime}$, where 
$\left(V^{\prime}, E^{\prime}\right):=\operatorname{tr}_{r}(u, \operatorname{tree}(u, I), T)$. Note that $\phi(u)$ is well-defined whenever, tree $(u, I)$ is a subgraph of $T$.

Lemma 15. Assume that invariant $\mathrm{H} 1$ and $\mathrm{H} 2$ holds at the beginning of the current iteration. Then, let $\mathcal{W}$ be a partitioning of $R \backslash\{r\}$ as described in Lemma 14. Further, let $(u, I)$ be the tuple that is chosen from $Q$ in the current iteration in Line 9 of Listing 2. If $(u, I)$ is optimal and $I \in \mathcal{W}$, then invariant $\mathrm{H} 2$ holds at the end of the current iteration as well.

Proof. In the following, we show that after the iteration there always exists a tuple $(v, I)$ such that H2.1 or H2.2 holds. To this end, let $T \in \operatorname{smt}(\mathcal{I})$ be any SMT containing tree $(u, I)$ as a subgraph. Due to optimality of $(u, I)$, such a graph exists. Then, let $S_{u, r}$ be the unique path from $u$ to $r$ in $T$.

We prove the lemma by contradiction. We therefore assume that no tuple $(v, I)$ exists, such that $H 2$ holds. Then, for all optimal tuples $\left(v^{\prime}, I\right) \in P$ we have that, $\left(v^{\prime}, I\right) \notin Q$ and $\left|\phi\left(v^{\prime}\right)\right|=0$. Next, we aim to arrive at a contradiction by induction on $\ell$, where $x_{\ell}$ denotes the $\ell$-th vertex in $S_{u, r}$.

- Induction Hypothesis: For every vertex $v^{\prime}$ of $S_{u, r}$ it holds that, $\left(v^{\prime}, I\right)$ is optimal, $\left(v^{\prime}, I\right) \in P,\left(v^{\prime}, I\right) \notin Q$, and $\left|\phi\left(v^{\prime}\right)\right|=0$.

- Base Case $\left(x_{1}\right):(u, I)$ is by definition optimal. In the current iteration $(u, I)$ is removed from $Q$ and added to $P$. Furthermore, by assumption we already have that $|\phi(u)|=0$.

- Induction Step $\left(x_{\ell+1}\right)$ : For vertex $x_{\ell+1}$ it holds by hypothesis that, $\left|\phi\left(x_{\ell}\right)\right|=0,\left(x_{\ell}, I\right) \notin Q,\left(x_{\ell}, I\right) \in P$ and $\left(x_{\ell}, I\right)$ is optimal. As a result, $\left(x_{\ell}, I\right)$ has been expanded with optimal costs. Then, $\left|\phi\left(x_{\ell}\right)\right|=0$ implies, that $x_{\ell}$ has degree at most two in $T$. If it had degree 3 or higher, one of the subgraphs would contain no terminal, contradicting that $T$ is minimal. Due to the optimality of $\left(x_{\ell}, I\right)$ and since $x_{\ell}$ has degree 1 or 2 in $T$, the optimal costs for $\left(x_{\ell+1}, I\right)$ are $l\left(x_{\ell}, I\right)+c_{N}\left(\left\{x_{\ell}, x_{\ell+1}\right\}\right)$. Therefore, after the expansion of $\left(x_{\ell}, I\right)$, the tuple $\left(x_{\ell+1}, I\right)$ was optimal. By R2.2, $\left(x_{\ell+1}, I\right)$ is therefore either in $Q$ or $P$. Then, by assumption $\left(x_{\ell+1}, I\right) \in P$ and also by assumption $\left|\phi\left(x_{\ell+1}\right)\right|=0$.

Since $r \in S_{u, r}$ and $|\phi(r)|>0$, we have a contradiction.

We now have all the tools to prove Lemma 14 . 
of Lemma 14. We show that the invariant holds at the beginning of the first iteration. Every tuple in $Q$ is of the form $(z,\{z\})$, where $z \in R \backslash\{r\}$. For each $z \in R \backslash\{r\}$, these sets $\{z\}$ induce a partition and $\operatorname{tree}(z,\{z\})$ is a subgraph of any SMT of $(N, R)$. As a result, $H 1$ and H2 sustains.

We assume that we are at the beginning of an arbitrary iteration, and assume that the invariant holds for the previous iteration. Next, we remove a tuple $(u, I)$ from $Q$. Towards showing the invariant for the current iteration, the proof proceeds by a case distinction on $(u, I)$.

Case 1: $(u, I)$ is not optimal. In this iteration, no optimal value can be "overwritten" in $l$, as the weight of any tuple processed in this iteration cannot be lower than an optimal value. As a result, the invariant still holds at the end of the iteration.

Case 2: $(u, I)$ is optimal, but there exists no partitioning $\mathcal{W}$, such that $I \in \mathcal{W}$ and $H 1$ and $H_{2}$ hold for $\mathcal{W}$. However, by the invariant of the previous iteration, a partition $\mathcal{W}$ exists such that $I \notin \mathcal{W}$. We show in the following, that neither loop in Line 17, nor loop in Line 12 invalidate the invariant.

Observe, that the loop in Line 17 cannot overwrite tentative cost values of any tuples used in the invariant. In particular, no optimal value in $l$ can be overwritten, and no changes to $l$ and $b$ are performed in case of equality.

Assume that the loop in Line 12 creates an optimal tuple $(u, I \cup J)$. This could invalidate the invariant only, if there exist $I^{\prime}, J^{\prime} \in \mathcal{W}$ such that $I \cup J=I^{\prime} \cup J^{\prime}$. Let $T$ be the SMT as defined in $H 1$. Then, $I \notin \mathcal{W}$ and therefore, either $I \nsubseteq I^{\prime}$ or $J \nsubseteq J^{\prime}$. This implies that either tree $(u, I)$ or tree $(u, J)$ is not a subraph of $T$. Therefore, H1.2 still holds, as well as the other invariants.

Case 3: $(u, I)$ is optimal and there exists a partitioning $\mathcal{W}$ as described in the lemma. We only consider optimal tuples that are created in $Q$ or $P$ during the iteration. All other tuples do not affect any of the invariants.

If no new optimal tuples in $Q$ are created, then, since invariant H2 was $^{2}$ true at the end of the previous iteration by Lemma 15, invariant H2 is still preserved. As $(u, I)$ is added to $P, H 1.1$ holds. And as no new tuples are created, H1.2 holds as well.

For every optimal tuple created in the loop at Line 17 (propagation), obviously H2.1 and therefore H2 is preserved. Since $(u, I)$ is added to $P$, H1.1 is also preserved. Finally, since all tuples are created using set $I, H 1.2$ holds as well.

For every optimal tuple processed in the loop at Line 12 (combination). Tuples created consist of set $I \cup J$. We proceed with a case distinction on $J$ : 
Case 3a: $J \notin \mathcal{W}$; $H 1$ holds after the iteration as $(u, I) \in P$. H1.1 holds since $(u, I) \in P$ and $H 1.2$ holds because $J \notin \mathcal{W}$. Since $(u, I \cup J)$ is optimal, there exists $T \in \operatorname{smt}(\mathcal{I})$ such that $\operatorname{tree}(u, I \cup J)$, $\operatorname{tree}(u, I)$ and tree $(u, J)$ are subgraphs of $T$. Since tree $(u, I)$ and tree $(u, J)$ are subgraphs of tree $(u, I \cup J)$, and each contains at least one terminal (since $I, J \neq \emptyset$ ), H2.2 holds.

Case 3b: $J \in \mathcal{W}$; We argue that $\mathcal{W}^{\prime}=(\mathcal{W} \backslash\{I, J\}) \cup\{I \cup J\}$ is a partition preserving the invariant. Let $T$ be the SMT as described in $H 1$ for $\mathcal{W}$. H1.1 holds since either tree $(u, I \cup J)$ is a subgraph of $T$, or one of tree $(u, I)$, tree $(u, J)$ is no subgraph of $T$. H1.2 sustains since it holds for the tuples $(u, I),(u, J)$ and therefore also for the tuple $(u, I \cup J)$. Since at the beginning of the iteration $(u, I \cup J) \notin P \cup Q$ by H1.2, $(u, I \cup J)$ is added to $Q$ and therefore 2.1 and consequently H2 holds.

Lemma 16. Whenever an element from $Q$ is chosen in the loop in Line 9 of Listing 2, there exists $(u, I) \in Q$ that is optimal.

Proof. Let $\mathcal{W}$ be a partition as described in Lemma 14. We first show the following Claim (1) that given $T$ as defined in $H 1$, we have that for every $W \in \mathcal{W}$, there exists an optimal tuple $(u, W) \in P$ such that $|\phi(u)|>0$ and tree $(u, W)$ is a subgraph of $T$. Let therefore $W \in \mathcal{W}$ be an arbitrary element and $(u, W)$ be an optimal tuple such that tree $(u, W)$ is a subgraph of $T$. By H1, such a tuple exists. Then, we establish H2.2 for tuple $(u, W)$, by using the same proof as in Lemma 15, but for specific tuple $(u, W)$. This concludes Claim (1).

Next, we prove the lemma by contradiction. We therefore assume that no tuple $(u, I) \in Q$ exists that is optimal. From Claim (1) above, we follow that for every $W \in \mathcal{W}$, there exists a tuple, satisfying H2.2, which is a subgraph of $T$. As each of the tuples defines a subgraph in $T$, we have $|\mathcal{W}|$ many subgraphs. There can be at most $|\mathcal{W}|-1$ many join vertices joining these subgraphs, since $T$ is a tree. We define for any $u \in V$ the set $A(u)=\{W \in \mathcal{W} \mid(u, W)$ is optimal $\}$ of sets in $\mathcal{W}$ constituting optimal tuples. Since we have $|\mathcal{W}|$ many subgraphs connected by at most $|\mathcal{W}|-1$ join vertices, there exists $u \in V$ such that $|A(u)|>1$ by basic combinatorics. Since all tuples $(u, W)$ with $W \in A(u)$ are optimal, the tuple $\left(u, \bigcup_{W \in A(u)} W\right)$ would have been added to $Q$ with optimal value in Line 16 . This contradicts H1.2. 
We are in the situation to prove our main result, Theorem 9 .

Theorem 17. Given an instance $(N, R)$ of $S T P$, where $N=(V, E, \sigma)$, and a root $r \in R$. Then, Algorithm DS*, cf., Listing 2, (1) terminates if $h^{*}$ is admissible. Further, after termination, (2) $l(r, R \backslash\{r\})$ is the cost of an SMT for the instance $(N, R)$, i.e., $l(r, R \backslash\{r\})=l^{*}(r, R \backslash\{r\}=\operatorname{csmt}(N, R)$.

Proof. For showing termination (1), consider that any value $l(u, I)$ that is set is clearly bounded above by the sum of all edges. Assuming $n:=|V|$, the Steiner network has $2^{n} \cdot n$ tuples. Since creating a new tuple $(u, I)$ in $Q$ requires that the found cost is lower than $l(u, I)$, therefore only $2^{n} \cdot n \cdot c_{N}((V, E))$ tuples can be created. Since this number is finite, the algorithm terminates.

For establishing correctness (2), we have to show that whenever $(r, R \backslash$ $\{r\}) \in P$, then $l(r, R \backslash\{r\})=\operatorname{csmt}(N, R)$. Towards a proof by contradiction, we assume that $(r, R \backslash\{r\}) \in P$ and $l(r, R \backslash\{r\})$ is not equal to the optimal weight. Since Steiner heuristic function $h^{*}$ is admissible, we have $h^{*}(v, R \backslash I) \leq$ $l^{*}(v, R \backslash I)$ for all $v \in V,\{r\} \subseteq I \subseteq R$. Let $w=\operatorname{csmt}(N, R)$. Due to the admissibility of $h^{*}$, it holds that $w^{\prime}:=l(r, R \backslash\{r\})+h^{*}(r,\{r\})=l(r, R \backslash\{r\})$. Since $(r, R \backslash\{r\})$ is not optimal by assumption, $w^{\prime}>w$. Since tuple $(r, R \backslash\{r\})$ was expanded, it holds that at this iteration, no tuple $(u, I) \in Q$ such that $l(u, I)+h^{*}(u, R \backslash I) \leq w$ existed. Any optimal tuple $(v, J)$ would have $l(v, J)+h^{*}(v, R \backslash J) \leq l^{*}(v, J)+l^{*}(v, R \backslash J)=w$ due to optimality and the admissibility of the Steiner heuristic function. Therefore, there existed no optimal tuple in $Q$, contradicting Lemma 16.

\section{Details about Preprocessing in $\mathbf{D S}^{*}$ Solve}

In this section, we give a description of all the preprocessing techniques mentioned in Section 4.2. We first introduce some concepts used in preprocessing and then discuss the different preprocessing operations. We assume in the following an instance $\mathcal{I}=(N, R)$, where $N=(V, E, \sigma)$, and $G=(V, E)$.

New Edges. It is common that a reduction introduces an edge $e$ with edge $\operatorname{costs} c_{e}$. Whenever $e$ is already part of $N$ we then retain the minimum of the costs, i.e., $\sigma(e) \leftarrow \min \left\{c_{e}, \sigma(e)\right\}$. Otherwise, we set $\sigma(e):=c_{e}$.

Contraction. An edge $\{u, v\}$ is contracted by performing the following steps: 
1. For each edge $\left\{v, v^{\prime}\right\} \in \delta_{G}(v) \backslash\{u, v\}$, add edge $\left\{u, v^{\prime}\right\}$ to $N$, with edge $\operatorname{costs} \sigma\left(\left\{v, v^{\prime}\right\}\right)$.

2. If $v \in R$ then $R \leftarrow(R \backslash\{v\}) \cup\{u\}$.

3. Remove vertex $v$ and edges $\delta_{G}(v)$ from $N$.

Bottleneck Steiner distance. Given a path $P$ between two vertices $u$ and $v$. Then, this path can be transformed into one or more elementary paths by splitting it at the intermediate terminals along $P$. The maximum $\operatorname{costs} c_{N}\left(P^{\prime}\right)$ among each $P^{\prime}$ of these elementary paths is the Steiner distance.

The bottleneck Steiner distance between vertices $u$ and $v$, denoted by $s_{\mathcal{I}}(u, v)$, is the minimum Steiner distance among all paths connecting $u$ and $v$. The restricted bottleneck Steiner distance $\bar{s}_{\mathcal{I}}(u, v)$ is the minimum Steiner distance among all paths connecting $u$ and $v$ without using the edge $\{u, v\}$. Observe that if $\{u, v\} \notin E$ then $\bar{s}_{\mathcal{I}}(u, v)=s_{\mathcal{I}}(u, v)[10$.

The values for $s_{\mathcal{I}}$ and $\bar{s}_{\mathcal{I}}$ can be computed exactly [21] or approximated [9. 33]. As computing the exact values might not pay off for large instances, we oftentimes use different approximations.

\section{D.1 Preprocessing Operations.}

The DS Solve solver uses a collection of preprocessing operations, as listed below in three categories.

1. Simple operations are fast and can therefore be run, whenever the instance is simplified.

(a) non-terminals of degree $\leq 1$ 21, Chapter 2.1.1]

(b) non-terminals of degree 2 [21, Chapter 2.2.2]

(c) terminals of degree 1 [21, Chapter 2.2.1]

(d) Include minimum terminal edge [21, Chapter 2.2.2]

2. Exclusions, which try to remove a sub-graph by showing that there exists an SMT without the sub-graph.

(a) Long Edges [33, Lemma 20]

(b) Steiner Distance [10, Chapter 3] 
(c) Non-Terminals of degree $k$ [10, Chapter 4]

(d) Dual ascent bounds [33, Chapter 3.4.2]

3. Inclusions try to merge a sub-graph with an other sub-graph by showing that there exists an SMT containing this sub-graph.

(a) Short Links [33, Lemma 23]

(b) Nearest Vertex 21, Chapter 2.2.3]

The preprocessing operations are run in the order presented above, where the simple operations are also applied in-between. For each operation, we define a threshold in terms of a vertex or edge ratio. Whenever an operation fails to remove the number of vertices or edges defined by its threshold, it is deactivated. As soon as no active operations are left, the instance is passed on to the solving algorithm. The thresholds avoid expensive computations with negligible results.

We discuss the different preprocessing operations used in our solver. Every preprocessing operation consists of a lemma indicating a "test" of applicability presented, and a transformation. Whenever the test identifies a vertex or edge, the transformation can be performed. After obtaining an SMT for the resulting preprocessed instance, these transformations are later reversed on the SMT. This yields a solution for the original instance.

Remove Non-Terminals of degree 0 or 1 . Finding non-terminal leaf vertices, which can be removed from $N$, can be done extremely fast.

Lemma 18. [21, Chapter 2.1.1] No vertex $u \in V, u \notin R$ of degree 1 or 0 can be in a Steiner minimal tree.

Connect Neighbors of Non-Terminals of degree 2. This operation makes use of an important property of non-terminals of degree two. Whenever any such vertex is in an SMT, both its neighbors have to be as well. Nonterminals of degree two can thereby be replaced by an edge connecting the two neighbors, as defined in the lemma.

Lemma 19. [21, Chapter 2.2.2] Given a vertex $u \in V, u \notin R$ of degree two. Let $\{u, v\},\left\{u, v^{\prime}\right\}$ be the two incident edges. Let $N^{\prime}$ be the network obtained by removing $u$ and adding edge $\left\{v^{\prime}, v\right\}$ with costs $\sigma(\{u, v\})+\sigma\left(\left\{u, v^{\prime}\right\}\right)$. Then, $\operatorname{csmt}(N, R)=\operatorname{csmt}\left(N^{\prime}, R\right)$. If $N$ already contains $\left\{v, v^{\prime}\right\}$ with $\sigma(\{u, v\})+$ $\sigma\left(\left\{u, v^{\prime}\right\}\right) \geq \sigma\left(\left\{v, v^{\prime}\right\}\right)$, then $u$ can be removed. 
Include Edge of Terminals of degree 1. This simple operation allows us to quickly identify edges that must be in any SMT and can therefore be contracted. This similarly also applies to edges of cost 0 .

Lemma 20. [21, Chapter 2.2.1] For any terminal $z \in R$ of degree one, the incident edge $\{z, u\}$ is in every SMT.

Include Minimum Terminal Edge. The following lemma is a generalization of the previous lemma and allows for more edges to be contracted.

Lemma 21. [21, Chapter 2.2.2] If the nearest vertex incident to a terminal $z$ is also a terminal $z^{\prime}$, the edge $\left\{z, z^{\prime}\right\}$ is in at least one SMT.

Steiner Distance. This operation makes use of the previously introduced bottleneck Steiner distance to identify removable edges. We perform it with different approximation methods for $s_{\mathcal{I}}$ and $\bar{s}_{\mathcal{I}}$.

Lemma 22. [10, Chapter 3] Any edge $\{u, v\}$ with $\sigma(\{u, v\})>s_{\mathcal{I}}(u, v)$ can be removed from $N$. The same holds for any edge $\{u, v\}$ such that $\sigma(\{u, v\}) \geq \bar{s}_{\mathcal{I}}(u, v)$ [33].

Remove Long Edges. This operation complements the previous one by considering removable edges that may have been missed.

Lemma 23. [33, Lemma 20] Let $M$ be the MST for the distance network $D_{N}(R)$. Furthermore, let cmax be the maximum cost among all edges in $M$. Any edge $\{u, v\}$ with $\sigma(\{u, v\})>$ cmax can be removed from $N$.

Remove Non-Terminals of degree $k$. The goal of this operation is to identify non-terminals, that cannot have a degree higher than two in an SMT. To this end, given a set $S \subseteq V$ of vertices, we define the Steiner distance network $D^{\prime}(V):=\left(V, V \times V, s^{\prime}\right)$, where $s^{\prime}(\{u, v\}):=s_{\mathcal{I}}(u, v)$. Further, let $M^{\prime}(V)$ be the MST of $D^{\prime}(V)$, and $c_{M^{\prime}}^{\prime}(V):=c_{D^{\prime}(V)}\left(M^{\prime}(V)\right)$ be the sum of edge costs of $M^{\prime}(V)$.

Lemma 24. [10, Chapter 4] For $u \in V \backslash R$ with $\left|\delta_{G}(u)\right| \geq 3$ exists an SMT where $u$ has maximum degree two if: For every $A \subseteq \delta_{G}(u)$ with $|A| \geq 3$, it holds that

$$
\sum_{\{u, v\} \in A} \sigma(\{u, v\}) \geq c_{M^{\prime}}^{\prime}(\{v \mid\{u, v\} \in A\})
$$


Given such a vertex $u$ and its neighbors $V_{A}=\left\{v \mid\{u, v\} \in \delta_{G}(u)\right\}$, we can remove $u$ and replace it by edges connecting its neighbors. We add edges $\left\{\left(v, v^{\prime}\right) \in V_{A} \times V_{A} \mid v \neq v^{\prime}\right\}$ with $\operatorname{costs} \sigma\left(\left\{v, v^{\prime}\right\}\right):=\sigma(\{u, v\})+\sigma\left(\left\{u, v^{\prime}\right\}\right)$ for $v, v^{\prime} \in V_{A}, v \neq v^{\prime}$.

Dual ascent bounds. We already discussed the dual ascent algorithm in Appendix B.1. Besides a lower bound $\tilde{w}$, the algorithm also maintains reduced arc costs $\tilde{\sigma}$. We discuss how to use these results to establish local lower bounds. Let $r$ denote the terminal that is designated as root, and let $R^{\prime}:=R \backslash\{r\}$.

Lemma 25. [33, Chapter 3.4.2] Let $\tilde{w}$ be the lower bound and $\tilde{c}$ be the reduced edge costs after a dual ascent run. Furthermore, let $\tilde{d}$ be the distance function using the reduced edge costs and directed paths. Given a vertex $u \in V$,

$$
\tilde{w}+\tilde{d}(r, u)+\min _{z \in R^{\prime}} \tilde{d}(u, z)
$$

is a lower bound on any Steiner tree containing $u$.

A similar bound can be derived for any edge $\{u, v\} \in E$. Let

$$
\begin{aligned}
& c_{u}=\tilde{d}(r, u)+\tilde{\sigma}((u, v))+\min _{z \in R^{\prime}} \tilde{d}(v, z) \\
& c_{v}=\tilde{d}(r, v)+\tilde{\sigma}((v, u))+\min _{z \in R^{\prime}} \tilde{d}(u, z)
\end{aligned}
$$

$\tilde{w}+\min \left\{c_{u}, c_{v}\right\}$ is a lower bound for any Steiner tree containing $\{u, v\}$.

Any vertex and edge, where this lower bound exceeds an upper bound can be removed from $N$. The upper bound can be computed using RSPH.

Short Links. This operation uses the concept of Voronoi regions. A Voronoi partitioning for $R$, separates the vertices of $N$ into $|R|$ disjoint sets, the Voronoi regions. For each terminal $z \in R$ we define the neighborhood $B(z)$, as those vertices $u \in V$ closer to $z$ than to any other terminal [33]. Ties are assumed to be broken randomly. More precisely

$$
u \in B(z) \Rightarrow d_{N}(u, z) \leq d_{N}\left(u, z^{\prime}\right) \text { for all } z^{\prime} \in R
$$

We say base $(u)=z$ iff $u \in B(z)$. Links are edges that connect two Voronoi regions, i.e., an edge $\{u, v\} \in E$ is a link iff base $(u) \neq \operatorname{base}(v)$ [33].

Any link identified by the following lemma, is a contractible edge. 
Lemma 26. [33, Lemma 23] Given a terminal $z$ and the two shortest links of its Voronoi region $\{u, v\},\left\{u^{\prime}, v^{\prime}\right\}$ such that base $(u)=\operatorname{base}\left(u^{\prime}\right)=z .\{u, v\}$ belongs to at least one SMT if

$$
\sigma\left(\left\{u^{\prime}, v^{\prime}\right\}\right) \geq d_{N}(z, u)+\sigma(\{u, v\})+d_{N}(v, \operatorname{base}(v))
$$

Nearest Vertex. This operation tries to find contractible edges incident to terminals. The idea is the following. If the shortest edge is short enough compared to the other edges, it is in an SMT.

Lemma 27. [21, Chapter 2.2.3] Let $z$ be a terminal with degree at least 2. Let $e^{\prime}=\{z, u\}, e^{\prime \prime}=\{z, v\}$ be a shortest and second shortest edge incident to $z$. There exists at least one SMT containing $e^{\prime}$, if there exists a terminal $z^{\prime}$ such that $z^{\prime} \neq z$ and

$$
\sigma\left(e^{\prime \prime}\right) \geq \sigma\left(e^{\prime}\right)+d_{N}\left(u, z^{\prime}\right)
$$

In case the lemma fails to identify certain edges, we can extend it. If we can show that the condition holds for any edge that connects $v$, this is equal to showing it for $e^{\prime \prime}$ :

Lemma 28. [34, Lemma 8] Given the definitions of the previous lemma, there exists at least one SMT containing $e^{\prime}$ if:

$$
\sigma(e) \geq \sigma\left(e^{\prime}\right)+d_{N}\left(u, z^{\prime}\right) \quad \text { for all } e \in \delta_{G}(z) \cup \delta_{G}(v) \backslash\left\{e^{\prime}, e^{\prime \prime}\right\}
$$

